\title{
Estabilidade de Liapunov e derivada radial
}

\author{
Gerard John Alva Morales
}

TESE APRESENTADA

$\mathrm{AO}$

INSTITUTO DE MATEMÁTICA E ESTATÍSTICA

DA

UNIVERSIDADE DE SÃO PAULO

PARA

OBTENÇÃO DO TÍTULO

$\mathrm{DE}$

DOUTOR EM CIÊNCIAS

Programa: Matemática Aplicada

Orientador: Prof. Dr. Manuel Valentim de Pera Garcia

Durante o desenvolvimento deste trabalho o autor recebeu auxílio financeiro do CNPq.

São Paulo, 31 de Outubro de 2014 


\title{
Estabilidade de Liapunov e derivada radial
}

\author{
Esta tese corresponde à redação final devidamente \\ corrigida e defendida por Gerard John Alva Morales, \\ e aprovada pela Banca Examinadora.
}

São Paulo, 31 de Outubro de 2014

Banca Examinadora:

Prof. Dr. Manuel Valentim de Pera Garcia (Orientador) -IME-USP

Prof. Dr. Fábio Armando Tal -IME-USP

Prof. Dr. Ricardo dos Santos Freire Jr. -IME-USP

Prof. Dr. Ricardo Miranda Martins -IMECC-UNICAMP

Prof. Dr. Fábio dos Santos -UFS 
Dedico este trabalho a:

Isadora, Adriana, Marco, Georgina e Miriam. 


\section{Agradecimentos}

Agradeço:

A minha família, pelo apoio moral, pelos ensinamentos, pelo carinho, pela confiança e pela força incondicional que me proporcionaram em todo momento; em particular durante este período no doutorado. Meu carinho e especial consideração para: Isadora Silva Alva Morales, Adriana Maria Silva Morales, Marco Ascención Alva Castillo, Mercedes Georgina Morales de Alva e Miriam Edith Alva Morales.

A meu orientador, Prof. Dr. Manuel Valentim de Pera Garcia, pela aceitação como seu aluno de doutorado no programa de matemática aplicada em momentos críticos na minha formação académica, pela generosidade em compartilhar comigo a matemática sutil que ele estuda, pela orientação e pelas valiosas sugestões que tornaram possível esta tese. À Profa. Dra. Sônia Regina Garcia, pelas correções do português e sugestões que deram maior estética a este trabalho.

As sugestões da banca examinadora durante a defesa de esta tese o qual permitiu considerável melhoramento na redação da mesma.

Aos amigos, Prof. Dr. Jorge Manuel Sotomayor Tello, pela camaradagem proporcionada em momentos de descontração no IME, FEA-USP durante a homenagem à Profa. Dra. Marilda Sotomayor e pelas conversações sobre equações integro-diferenciais singulares, Prof. Dr. Alexandre Patriota Galvão, pelas conversações de caracter filosófico durante repetidas tardes de café, Dr. Pedro Losco Takecian, pela cordialidade e pelas sugestões técnicas sobre Ubuntu, ambiente onde preparei esta tese.

A hospitalidade dos departamentos de matemática e matemática aplicada do IME-USP que facilitaram o desenvolvimento desta tese, e o auxílio financeiro do CNPq.

Extensivos agradecimentos a todas e cada uma das pessoas que contribuíram à concretização deste trabalho, particulares considerações para José da Silva, Teodora da Silva, Ana, Paul Lopez, Madalena e Bispo. 


\section{Resumo}

Apresentaremos uma classe de energias potenciais $\Pi \in C^{\infty}(\Omega, \mathbb{R})$ que são $s$-decidíveis e que admitem funções auxiliares de Četaev da forma $\left\langle\nabla j^{s} \Pi(q), q\right\rangle, q \in \Omega \subset \mathbb{R}^{n}$ que são $s$-resistentes.

Palavras-chave: Estabilidade de Liapunov, sistemas Lagrangeanos, Teorema de Dirichlet-Lagrange, k-decidibilidade. 


\section{Abstract}

We will present a class of potential energies $\Pi \in C^{\infty}(\Omega, \mathbb{R})$ that are $s$-decidable and that admit auxiliary functions of Cetaev of the form $\left\langle\nabla j^{s} \Pi(q), q\right\rangle$, $q \in \Omega \subset \mathbb{R}^{n}$ which are $s$-resistant.

Keywords: Liapunov stability, Lagrangian systems, Theorem of DirichletLagrange, k-decidability. 


\section{Conteúdo}

$\begin{array}{llr}1 & \text { Introdução } & 9\end{array}$

2 Preliminares, o problema e um lema 13

2.1 Funções $s$-decidíveis . . . . . . . . . . . . . . . . 13

2.2 Formulação do problema . . . . . . . . . . . . . . . . . 14

2.2 .1 O problema . . . . . . . . . . . 16

2.3 Lema fundamental . . . . . . . . . . . . . . . . 17

3 Cones tangentes e $s$-resistência $\quad 21$

3.1 O cone $Z_{s-1} \ldots \ldots \ldots \ldots \ldots \ldots \ldots \ldots$

3.2 Construindo o cone $\mathcal{K}_{s} \ldots \ldots \ldots \ldots \ldots \ldots \ldots$

$3.3 s$-resistência de $P_{s} \ldots \ldots \ldots \ldots \ldots \ldots$

4 Aplicações da $s$-resistência de $P_{s} \quad 39$

4.1 Instabilidade do equilíbrio segundo Liapunov . . . . . . . . . 39

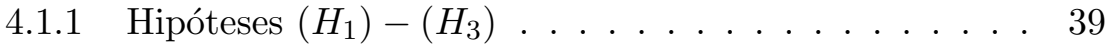

4.1.2 Hipóteses $\left(H_{1}^{1}\right)-\left(H_{3}\right) \ldots \ldots \ldots \ldots$. . . . . . 40

4.1.3 Hipóteses $\left(H_{1}^{2}\right)-\left(H_{3}\right) \ldots \ldots \ldots \ldots$. . . . . . . . . . . . . . . 41

4.2 Análise da hipótese $\left(H_{1}\right) \ldots \ldots \ldots$. . . . . . . . . . . . . . . . . . . . . . . . . . 42

4.3 Existência de trajetórias assintóticas . . . . . . . . . . 46 


\section{Capítulo 1}

\section{Introdução}

Neste trabalho, estamos interessados em estudar a estabilidade segundo Liapunov de um equilíbrio $\left(q_{0}, p_{0}\right) \in \mathbb{R}^{n} \times \mathbb{R}^{n}$ do sistema mecânico com $n$ graus de liberdade, cuja dinâmica é governada pelo sistema hamiltoniano

$$
X_{H}(q, p)\left\{\begin{array}{l}
\dot{q}=\frac{\partial H}{\partial p}(q, p) \\
\dot{p}=-\frac{\partial H}{\partial q}(q, p)
\end{array}\right.
$$

com hamiltoniana $H(q, p)=T(q, p)+\Pi(q),(q, p) \in \Omega \times \mathbb{R}^{n}$, onde $\Omega \subset \mathbb{R}^{n}$ é uma vizinhança aberta de $q_{0}$. Admitiremos que a energia cinética $T(q, p)$ é uma forma quadrática definida positiva na variável $p$, assim, podemos ver que os equilíbrios deste sistema, isto é, os pontos tais que $X_{H}\left(q_{0}, p_{0}\right)=(0,0)$, são os pontos $\left(q_{0}, 0\right)$, em que $q_{0}$ é um ponto crítico de $\Pi$.

O teorema de Dirichlet-Lagrange afirma que, se em $q_{0}$ a energia potencial $\Pi$ tem um mínimo estrito local, então o equilíbrio $\left(q_{0}, 0\right)$ do sistema $X_{H}$ é estável.

O seguinte exemplo com 1 grau de liberdade com hamiltoniana definida em $\mathbb{R} \times \mathbb{R}$

$$
T(q, p)=\frac{1}{2} p^{2}, \quad \Pi(q)= \begin{cases}e^{-1 / q^{2}} \cos (1 / q) & , \quad q \neq 0 \\ 0 & , \quad q=0\end{cases}
$$

mostra que a recíproca do teorema de Dirichlet-Lagrange é falsa, pois neste caso a origem $\left(q_{0}, 0\right)=(0,0)$ é estável mas $\Pi(q)$ não tem mínimo em $q_{0}=0$. Apesar deste exemplo, a situação de sistemas com um grau de liberdade $(n=1)$ é a única em que a estabilidade do equilíbrio é caracterizada de forma completa por propriedades da energia potencial. 
De fato, se $n=1$, a origem $(0,0)$ é estável segundo Liapunov se, e só se, existem sequências $\left(q_{n}^{+}\right)$e $\left(q_{n}^{-}\right)$, convergentes para $0,\left(q_{n}^{+}\right)$estritamente decrescente, $\left(q_{n}^{-}\right)$estritamente crescente, tais que $\Pi\left(q_{n}^{+}\right)>0$ e $\Pi\left(q_{n}^{-}\right)>0$ (para uma demonstração veja $[\mathrm{LHR}]$ ).

Em termos topológicos, esta condição para $n=1$ pode ser reescrita assim, " para sistemas com um grau de liberdade, a origem $(0,0)$ é estável segundo Liapunov se, e só se, existe um sistema fundamental de vizinhanças da origem, $\left(U_{n}\right)$, tal que $\Pi(q)>0, \forall q \in \partial U_{n}$, para todo $n \in \mathbb{N}$ ".

Durante algum tempo acreditou-se que a condição acima caracterizasse a estabilidade da origem para o caso geral de sistemas com $n$ graus de liberdade, entretanto, embora esta seja efetivamente uma condição suficiente para a estabilidade da origem, ela não é necessária, conforme se vê pelo exemplo abaixo, devido a Laloy (1977), para o caso de dois graus de liberdade.

Considere em $\mathbb{R}^{2} \times \mathbb{R}^{2}, T(q, p)=\frac{1}{2}\left(p_{1}^{2}+p_{2}^{2}\right)$ e $\Pi(q)$ a função

$$
\Pi\left(q_{1}, q_{2}\right)= \begin{cases}e^{-\frac{1}{q_{1}^{2}}} \cos \left(\frac{1}{q_{1}}\right)-e^{-\frac{1}{q_{2}^{2}}}\left[q_{2}^{2}+\cos \left(\frac{1}{q_{2}}\right)\right] & , \quad q_{1} q_{2} \neq 0 \\ 0 & , \quad q_{1} q_{2}=0 .\end{cases}
$$

Note que, na verdade, $\Pi$ é de classe $C^{\infty}$. Aqui, o equilíbrio $\left(q_{0}, 0\right)=(0,0)$ do sistema $X_{H}$ é estável, mas na reta $\left\{q_{2}=q_{1}\right\}$, tem-se para $q_{1} \neq 0$, $\Pi\left(q_{1}, q_{1}\right)=-q_{1}^{2} e^{-1 / q_{1}^{2}}<0$.

Nos dois exemplos anteriores $\Pi$ é uma função $C^{\infty}$, mas não analítica, de fato, todas as derivadas de $\Pi$ em 0 anulam-se. No contexto de funções analíticas o problema da estabilidade de Liapunov de um equilíbrio está discutido em $[\mathrm{P}]$.

Neste trabalho estudamos um problema que na literatura (ver por exemplo [LHR]) é conhecido como inversão do teorema de Dirichlet-Lagrange e consiste em estabelecer condições suficientes sobre a energia potencial para nosso sistema $X_{H}$ que garantam a instabilidade do equilíbrio $\left(q_{0}, 0\right)=(0,0)$ deste sistema hamiltoniano.

Existem muitos trabalhos relevantes abordando este problema (por exemplo $[\mathrm{LP}],[\mathrm{MN}],[\mathrm{GT}]$ e $[\mathrm{FGT}]$ ), mas o problema ainda não tem uma completa solução para o caso geral de $n$ graus de liberdade.

Um dos primeiros resultados nesta direção foi mostrado pelo próprio A.M. Liapunov, e mostra que: se a matriz hessiana de $\Pi$ em $q_{0}$ mostra que $\Pi$ não tem mínimo nesse ponto, então $\left(q_{0}, 0\right)$ é um equilíbrio instável do sistema $X_{H}$. Utilizando a linguagem de jatos pontuais, introduzida por A. Barone em sua tese de livre docência (veja [B]) e que exporemos abaixo, este resultado de Liapunov pode ser reparafraseado como: se o jato pontual de 
ordem 2 de $\Pi$ em $q_{0}$ mostra que a energia potencial $\Pi$ não tem minimo em $q_{0}$, então o equilíbrio $\left(q_{0}, 0\right)$ do sistema hamiltoniano $X_{H}$ é instável.

$\mathrm{Na}$ classe de energias potenciais $\Pi$ que admitem jatos de orden $s \geq 2$ no ponto crítico $q_{0}$, a generalização natural deste resultado é a:

Conjectura (Liapunov-Barone)

Se para algum natural $s \geq 2$, o jato de ordem $s$ de $\Pi$ em $q_{0}$ mostra que a energia potencial $\Pi$ não tem mínimo em $q_{0}$, então o equilíbrio $\left(q_{0}, 0\right)$ do sistema hamiltoniano $X_{H}$ é instável.

No caso em que o jato de ordem $s$ de $\Pi$ é um polinômio homogêneo, no contexto de $n$ graus de liberdade, o artigo [MN] mostra, usando a teoria da variedade estável, que, o equilíbrio $\left(q_{0}, 0\right)$ do sistema $X_{H}$, é instável.

No artigo [GT] mostrou-se que esta conjectura é verdadeira no contexto de 2 graus de liberdade, e em [FGT] mostrou-se parcialmente, que a conjectura é verdadeira no contexto geral de $n$ graus de liberdade. Nestes dois resultados usaram-se funções auxiliares como ferramenta básica.

Tanto no trabalho $[\mathrm{MN}]$ como em $[\mathrm{GT}]$ e $[\mathrm{FGT}]$ demonstrou-se não apenas a instabilidade de $\left(q_{0}, 0\right)$, mas a existência de trajetórias assintóticas para esse equilíbrio.

Outro resultado clássico neste problema é um teorema de Četaev que garante a instabilidade de $\left(q_{0}, 0\right)$ se existe uma componente conexa do conjunto $\Pi^{-1}(-\infty, 0)$ aderente a $q_{0}$ em que a derivada radial de $\Pi,\langle\nabla \Pi(q), q\rangle$, é estritamente negativa (se $\Pi$ tem essa propriedade diremos que $\Pi$ é um potencial de tipo Četaev).

Neste trabalho consideramos uma questão que relaciona estas duas formas de abordar o problema da inversão do teorema de Dirichlet-Lagrange, mais precisamente, admitiremos que $j^{s} \Pi$ mostra que $q_{0}$ não é ponto de mínimo de $\Pi$ e que $j^{s} \Pi$ é um potencial de tipo Četaev (isto é, suporemos que a derivada radial de $j^{s} \Pi,\left\langle\nabla j^{s} \Pi(q), q\right\rangle$ é estritamente negativa numa componente conexa de $\left.\left(j^{s} \Pi\right)^{-1}(-\infty, 0)\right)$, e procuramos determinar situações em que isto garantisse que $\Pi$ é um potencial de tipo Četaev (e portanto a instabilidade de $\left(q_{0}, 0\right)$ fica provada).

Conseguimos assim determinar uma classe de energias potenciais em que a instabilidade da origem é garantida por jatos.

O resultado central do trabalho (veja o Teorema 4.1.1) afirma, em linguagem um pouco livre (para definições precisas veja a seção 2.1) que se a energia potencial $\Pi: \Omega \rightarrow \mathbb{R}, \Omega \subset \mathbb{R}^{n}$ aberto com $0 \in \Omega$, é suficientemente regular e tem um ponto crítico na origem, com $\Pi(0)=0$, satisfaz:

$\left(H_{1}\right)$ Para $\ell \leq s-1, j^{\ell} \Pi(q) \geq 0$ para $q$ numa vizinhança da origem.

$\left(H_{2}\right) j^{s} \Pi$ mostra que $\Pi$ não tem mínimo na origem. 
$\left(H_{3}\right)$ Existe $\varepsilon>0$ tal que

$$
\overline{\left\{q \in \mathbb{R}^{n}\left|j^{s} \Pi(q)<0,\right| q \mid<\varepsilon\right\}} \backslash\{0\} \subset\left\{q \in \mathbb{R}^{n}\left|\left\langle\nabla j^{s} \Pi(q), q\right\rangle<0,\right| q \mid<\varepsilon\right\} .
$$

Então $\Pi$ é um potencial de tipo Četaev.

Isso mostra que nos sistemas $X_{H}$ em que as energias potenciais satisfazem $\left(H_{1}\right),\left(H_{2}\right)$ e $\left(H_{3}\right)$ o ponto $(0,0)$ é instável segundo Liapunov, o que justifica nossa afirmação anterior sobre caracterizar uma classe de potenciais em que a instabilidade é garantida por jatos.

Os resultados obtidos neste trabalho garantem além da instabilidade da origem do sistema $X_{H}$, a existência de trajetórias assintóticas à origem de $X_{H}$.

As hipóteses $\left(H_{2}\right)$ e $\left(H_{3}\right)$ feitas sobre $\Pi$ são, em um sentido que expomos no texto, bastante naturais neste contexto. Já a hipótese $\left(H_{1}\right)$ parece, à primeira vista, um pouco restritiva demais, porém mostramos na seção 4.2 que, enfraquecendo um pouco essa hipótese, a conclusão do nosso resultado central não se mantem.

Organizamos nosso trabalho da seguinte forma: no capítulo 2 apresentamos notações e definições técnicas que serão de utilidade para depois colocar nosso problema assim como nossas hipóteses na classe supramensionada de energias potenciais $\Pi$. No capítulo 3 mostramos nosso resultado principal e no capítulo 4 mostramos algumas consequências e conclusões finais. 


\section{Capítulo 2}

\section{Preliminares, o problema e um lema}

O objetivo deste capítulo é apresentar nosso problema e também apresentar um lema que será fundamental no resto do trabalho.

\subsection{Funções $s$-decidíveis}

No espaço euclidiano $\left(\mathbb{R}^{n},|\cdot|\right)$ com a norma usual, consideramos uma vizinhança aberta da origem $\Omega$ e denotamos por $B\left(q_{0}, \varepsilon\right)$ o conjunto aberto

$$
B\left(q_{0}, \varepsilon\right)=\left\{q \in \mathbb{R}^{n}|\quad| q-q_{0} \mid<\varepsilon\right\} .
$$

Quando $q_{0}=0$, simplesmente denotaremos esta bola por $B_{\varepsilon}$.

Como é usual, para funções $f, g: \Omega \rightarrow \mathbb{R}$ em que $g(q) \neq 0$ se $q \neq 0$, a notação $f=o(g)$ em 0 significa que

$$
\lim _{q \rightarrow 0} \frac{f(q)}{g(q)}=0
$$

Definição 2.1.1 Sejam s um natural não nulo e $\Omega$ uma vizinhança aberta da origem de $\mathbb{R}^{n}$. Dizemos que uma função $f: \Omega \rightarrow \mathbb{R}$ tem jato pontual de ordem s na origem, se existe um polinômio de grau menor ou igual a $s$, que denotamos por $j^{s} f$, tal que

$$
f(q)=j^{s} f(q)+o\left(|q|^{s}\right) .
$$

Observação 2.1.2 É simples ver que existe no máximo um polinômio que satisfaz estas condições e, se f for de classe $C^{s}$, então $f$ tem jato pontual de ordem s na origem e $j^{s} f$ é o polinômio de Taylor de ordem s de $f$ em 0 . 
Denotemos por $J^{s}(\Omega, \mathbb{R})$ o conjunto das funções $f: \Omega \rightarrow \mathbb{R}$, com $f(0)=0$, que admitem jato pontual de ordem $s$ na origem.

Diremos que $f$ tem um mínimo forte (respectivamente um máximo forte) na origem se existe $\varepsilon>0$ tal que $f(x)>0$ (respectivamente $f(x)<0$ ) para todo $x \in(\Omega \backslash\{0\}) \cap B_{\varepsilon}$; diz-se que $f$ tem um mínimo fraco (respectivamente um máximo fraco) na origem se existe $\varepsilon>0$ tal que $f(x) \geq 0$ (respectivamente $f(x) \leq 0)$ para todo $x \in \Omega \cap B_{\varepsilon}$ e existe uma sequência $\left(x_{k}\right), x_{k} \neq 0$, com $x_{k} \rightarrow 0$ e $f\left(x_{k}\right)=0$.

Nestes casos diz-se que $f$ tem extremo forte, ou extremo fraco, na origem.

Se $f$ não tem extremo forte ou fraco na origem, diremos que $f$ tem sela na origem.

Diz-se que as funções $f: \Omega \rightarrow \mathbb{R}$ e $g: \Omega \rightarrow \mathbb{R}$ têm mesmo comportamento em relação a extremo na origem se $f$ e $g$ tiverem ambas mínimo forte (respectivamente máximo forte) na origem, ou ambas tiverem mínimo fraco (respectivamente máximo fraco) na origem, ou ambas tiverem sela na origem.

Definição 2.1.3 Uma função $f \in J^{s}(\Omega, \mathbb{R})$ é s-decidível se, para cada função $g \in J^{s}(\Omega, \mathbb{R})$ tal que $j^{s} f=j^{s} g$, tem-se que ambas as funções $f$ e g possuem o mesmo comportamento em relação a extremo na origem.

Denotamos por $D^{s}(\Omega, \mathbb{R})$ a classe de funções $f \in J^{s}(\Omega, \mathbb{R}) s$-decidíveis na origem. Uma consequência direta desta definição é que, se $f \in D^{s}(\Omega, \mathbb{R})$, então na origem $0, f$ tem extremo forte ou uma sela, conforme se vê na observação abaixo.

Observação 2.1.4 Se $f \in J^{r}(\Omega, \mathbb{R})$ e $j^{r} f$ tem na origem um extremo brando, então $f \notin D^{r}(\Omega, \mathbb{R})$. Para ver isto, basta supor, sem perda de generalidade que $j^{r} f$ tem mínimo fraco e considerar a função $g(q):=j^{r} f(q)+$ $|q|^{2 r} \in J^{r}(\Omega, \mathbb{R}) ;$ observemos que enquanto $j^{r} f$ tem minimo fraco, $g$ tem minimo forte na origem; e se considerarmos $g(q):=j^{r} f(q)-|q|^{2 r} \in J^{r}(\Omega, \mathbb{R})$, $g$ tem uma sela na origem (ou máximo, caso $f \equiv 0$ ).

Mais detalhes acerca de $s$-decidibilidade podem ser vistos em $[\mathrm{B}]$ ou $[\mathrm{G}]$.

\subsection{Formulação do problema}

Sejam dois números naturais $k$ e $s$, com $2 \leq k<s$, e consideremos funções $f \in J^{s}(\Omega, \mathbb{R})$ cujo primeiro jato não nulo é o de ordem k. Então o jato de ordem $s$ de $f$ na origem, pode ser escrito da seguinte forma

$$
j^{s} f(q)=f_{k}(q)+f_{k+1}(q)+\ldots+f_{s-1}(q)+f_{s}(q)
$$


onde, para cada $\ell \in\{k, \ldots, s\}, f_{\ell}(q)=j^{\ell} f(q)-j^{\ell-1} f(q)$. Note que $f_{\ell}$ é um polinômio $\ell$-homogêneo (isto é, $f_{\ell}(\lambda q)=\lambda^{\ell} f_{\ell}(q)$, para todo $\lambda \in \mathbb{R}$ e para todo $q \in \mathbb{R}^{n}$ ), chamada parte homogênea de grau $\ell$ de $j^{s} f$. Veja ainda que $f_{k}=j^{k} f$.

Estamos interessados em energias potenciais cujo jato de ordem $s$ mostra que esta função não têm mínimo na origem, mais precisamente isto é formalizado na definição abaixo.

Definição 2.2.1 Dada uma função $f \in J^{s}(\Omega, \mathbb{R})$, dizemos que o jato $j^{s} f$ mostra que $f$ não tem mínimo na origem se, para cada função $g \in J^{s}(\Omega, \mathbb{R})$ com $j^{s} f=j^{s} g$, tem-se que $g$ não tem mínimo na origem.

Observação 2.2.2 Note que o fato de $j^{s} f$ mostrar que $f$ não tem mínimo na origem não implica que $f$ é s-decidivel, por exemplo, se $j^{2} f(x, y)=-x^{2}$, claro que esse jato mostra que $f$ não tem minimo na origem, mas $f$ não é 2-decidível, pois $j^{2} f$ tem máximo não estrito na origem. Entretanto se o primeiro jato não nulo de $f$ na origem tem mínimo fraco nesse ponto e $j^{s} f$ mostra que $f$ não tem mínimo na origem, é simples ver que $f$ é s-decidivel e tem sela na origem.

Será de fundamental importância estudar o comportamento da derivada radial de $j^{s} \Pi$ na origem, $P_{s}(q):=\left\langle\nabla j^{s} \Pi(q), q\right\rangle$; em geral, as funções $j^{s} \Pi$ e $P_{s}$ não têm o mesmo comportamento em 0 , como pode ser visto no seguinte exemplo

Exemplo 2.2.3 Consideremos um polinômio

$f\left(q_{1}, \bar{q}\right):=\left(a q_{1}-b|\bar{q}|^{2}\right)^{2}+c|\bar{q}|^{4}, \quad c o m \quad a>0, \quad b>0, \quad c>0, \quad b^{2}>8 c$.

e onde $\bar{q}:=\left(q_{2}, \ldots, q_{n}\right)$. Notemos que

$$
\begin{aligned}
\frac{\partial f}{\partial q_{1}} & =2 a\left(a q_{1}-b|\bar{q}|^{2}\right) \\
\frac{\partial f}{\partial q_{j}} & =\left[-4 a b q_{1}+\left(4 b^{2}+4 c\right)|\bar{q}|^{2}\right] q_{j}, \quad j=2, \ldots, n
\end{aligned}
$$

assim, a derivada radial $\langle\nabla f(q), q\rangle=\frac{\partial f}{\partial q_{1}} q_{1}+\sum_{j=2}^{n} \frac{\partial f}{\partial q_{j}} q_{j}$, escreve-se como

$$
\langle\nabla f(q), q\rangle=2 a^{2}\left(q_{1}-\frac{3 b}{2 a}|\bar{q}|^{2}\right)^{2}-\left(\frac{b^{2}-8 c}{2}\right)|\bar{q}|^{4} .
$$

É claro que na origem, $f(q)$ tem minimo forte e $\langle\nabla f(q), q\rangle$ tem uma sela. 
A função $\langle\nabla f(q), q\rangle$, foi utilizada por Četaev para mostrar o seguinte resultado de instabilidade

Teorema 2.2.4 (N.G. Četaev [1936])

Suponha que $\overline{B_{\varepsilon}} \subset \Omega$, para algum $\varepsilon>0$ e que o sistema hamiltoniano $X_{H}$ satisfaz as propriedades

1. $\Theta=\left\{q \in B_{\varepsilon} \mid \Pi(q)<0\right\} \neq \emptyset$

2. $0 \in \partial \Theta$

3. $\langle\nabla \Pi(q), q\rangle<0, \quad \forall q \in \Theta$

então o equilíbrio $(q, p)=(0,0)$ é instavel.

Uma demonstração deste resultado pode ser vista, por exemplo, em [LHR].

Se uma energia potencial $\Pi$ satisfaz as condições deste teorema diremos que $\Pi$ é um potencial do "tipo Čtaev".

O que faremos neste trabalho será determinar se $\Pi$ é um potencial do "tipo Četaev" a partir de propriedades de $j^{s} \Pi$. Mais precisamente, descrevemos este problema a seguir.

\subsubsection{O problema}

Supondo que $j^{s} \Pi$ é uma energia potencial do tipo Četaev, será verdade que $\Pi$ também é uma energia potencial do tipo Četaev?

Esta questão, sem outras hipóteses, tem uma resposta negativa, mesmo quando $j^{s} \Pi$ mostra que $\Pi$ não tem mínimo na origem, e um exemplo mostrando isto será apresentado em algum detalhe no capítulo 4 .

A fim de trabalhar no contexto das energias potenciais $\Pi \in J^{s}(\Omega, \mathbb{R})$ que não apresentam mínimo na origem, consideramos $P_{s}(q):=\left\langle\nabla j^{s} \Pi(q), q\right\rangle$ e os conjuntos semi-algébricos

$$
A_{s}:=\left\{q \in \Omega \mid j^{s} \Pi(q)<0\right\}, \quad C_{s}:=\left\{q \in \Omega \mid P_{s}(q)<0\right\}
$$

e vamos supor que a energia potencial $\Pi \in J^{s}(\Omega, \mathbb{R})$ satisfaz:

$\left(H_{1}\right) j^{\ell} \Pi(q) \geq 0$ numa vizinhança da origem $0 \in \Omega$, se $\ell \leq s-1$;

$\left(H_{2}\right) j^{s} \Pi$ mostra que $\Pi$ não tem mínimo na origem $0 \in \Omega$;

$\left(H_{3}\right)$ existe $\varepsilon>0$ com $\overline{B_{\varepsilon}} \subset \Omega$, tal que $\left(\bar{A}_{s} \backslash\{0\}\right) \cap B_{\varepsilon} \subset C_{s}^{\circ} \cap B_{\varepsilon}$. 
Claro que $\left(H_{3}\right)$ mostra que $j^{s} \Pi$ é um potencial do tipo Četaev.

Nosso principal resultado é:

\section{Teorema.}

"Se $\Pi$ satisfaz as hipóteses $\left(H_{1}\right)-\left(H_{3}\right)$, então $\Pi$ é um potencial de tipo Četaev".

Mostraremos também que, enfraquecendo um pouco a hipótese $\left(H_{1}\right)$ esse resultado deixa de ser verdadeiro.

\subsection{Lema fundamental}

Nesta seção colocamos em evidência a importância da hipótese $\left(H_{3}\right)$, o que será crucial na construção feita no próximo capítulo.

Se $\gamma:[0, \rho] \rightarrow \mathbb{R}^{n}$ é uma curva algébrica tal que $\gamma(0)=0$ e $\gamma(t) \neq 0$ para todo $t \in(0, \rho]$; denotamos por $r_{\gamma}$ a semi-reta tangente a $\gamma$ na origem. Para detalhes técnicos acerca de curvas algébricas e conjuntos semi-algébricos referimos a $[\mathrm{M}]$.

Lema 2.3.1 Suponha que $\Pi \in J^{s}(\Omega, \mathbb{R})$ satisfaz a hipótese $\left(H_{3}\right)$ e seja $\gamma$ : $[0, \rho] \rightarrow \mathbb{R}^{n}$ uma curva algébrica tal que $\gamma(0)=0$ e $\gamma(t) \in A_{s}$ se $t \in(0, \rho]$. Então $\left.j^{s} \Pi\right|_{r_{\gamma}}$ tem máximo local estrito na origem.

\section{DEMONSTRAÇÃo}

Seja $v \neq 0$ o versor de $\gamma$ em $0^{+}$. Como $j^{s} \Pi$ é um polinômio de grau $s$, existe $\varepsilon>0$ tal que uma das seguintes possibilidades acontece:

(a) $j^{s} \Pi(\lambda v)>0$, para todo $\lambda \in(0, \varepsilon)$

(b) $j^{s} \Pi(\lambda v)=0$, para todo $\lambda \in(0, \varepsilon)$

(c) $j^{s} \Pi(\lambda v)<0$, para todo $\lambda \in(0, \varepsilon)$

e $\lambda v, \lambda \in \mathbb{R}^{+}$, é uma parametrização de $r_{\gamma}$. Mostremos que as possibilidades (a) e (b) não ocorrem.

1. Suponha que $(a)$ ocorre. Tome $q=\frac{\varepsilon}{2} v$, então $j^{s} \Pi(q)>0$ e escolha $\delta>0$ tal que $j^{1} \Pi(x) \geq 0$, para todo $x$ tal que $|x-q|<\delta$. Seja agora $\Sigma_{\delta}(q)$ o disco de dimensão $n-1$ centrado em $q$ de raio $\delta$, ortogonal a $v$. Tomando então o tronco de cone $\Delta$ de vértice 0 e base $\Sigma_{\delta}(q)$, veja que, como $v$ é o versor de $\gamma$ em $0^{+}$e, para $t>0, \gamma(t) \in A_{s}$,

\footnotetext{
${ }^{1}$ Neste ponto pode-se garantir $j^{s} \Pi(x)>0$ em $B(q, \delta)$, preferimos a desigualdade branda pois assim faz-se um raciocínio que será usado mais adiante nesta demonstração.
} 
resulta que existe um ponto $p$ no interior de $\Delta$ tal que $j^{s} \Pi(p)<0$. Considere a semi-reta de origem 0 que passa por $p$ e note que, como $p$ está no interior de $\Delta$ existe exatamente um ponto $w$ dessa semi-reta que está em $\Sigma_{\delta}(q)$. Claro que $p$ é um ponto do interior do segmento $0 w$ e, como $j^{s} \Pi$ é um polinômio, $j^{s} \Pi(p)<0$ e $j^{s} \Pi(w) \geq 0$, resulta que existe $t_{u} \in(0,1)$ tal que o ponto $u:=t_{u} w$ do segmento $0 w$ satisfaz,

(i) $j^{s} \Pi(t w) \geq 0$, se $t_{u} \leq t \leq 1$;

(ii) existe $\rho>0$ tal que $j^{s} \Pi(t w)<0$, se $t_{u}-\rho<t<t_{u}$.

Portanto $u \in \bar{A}_{s} \backslash\{0\}$ e $\left.\frac{d}{d t} j^{s} \Pi(t w)\right|_{t=t_{u}} \geq 0$. Como

$$
\left.\frac{d}{d t} j^{s} \Pi(t w)\right|_{t=t_{u}}=\left\langle\nabla j^{s} \Pi\left(t_{u} w\right), w\right\rangle=\frac{1}{t_{u}} P_{s}(u)
$$

resulta $P_{s}(u) \geq 0$. Isso contraria a hipótese $\left(H_{3}\right)$, e mostra que $(a)$ não pode acontecer.

2. Suponha que $(b)$ ocorre. Tome outra vez $q=\frac{\varepsilon}{2} v$ e agora escolha $\Sigma_{1}(q)$ o disco de dimensão $n-1$ centrado em $q$ de raio 1 , ortogonal a $v$. Se existe uma sequência de pontos $\left(q_{k}\right) \subset \Sigma_{1}(q) \backslash\{q\}, q_{k} \rightarrow q$, tais que $j^{s} \Pi\left(q_{k}\right)<0$ então $q \in \overline{A_{s}} \backslash\{0\}$, e como $j^{s} \Pi(\lambda v)=0$, se $\lambda \in(0, \varepsilon)$, resulta

$$
P_{s}(q)=\frac{\varepsilon}{2}\left\langle\nabla j^{s} \Pi\left(\frac{\varepsilon}{2} v\right), v\right\rangle=\left.\frac{\varepsilon}{2} \frac{d}{d \lambda} j^{s} \Pi(\lambda v)\right|_{\lambda=\frac{\varepsilon}{2}}=0
$$

contrariando outra vez a hipótese $\left(H_{3}\right)$. Se isso não acontece, existe $\delta>0$ tal que $j^{s} \Pi(x) \geq 0$, para todo $x \in \Sigma_{\delta}(q)$, aplicamos o raciocinio do item $(a)$ contrariando a hipótese $\left(H_{3}\right)$ também neste caso. Assim, (c) ocorre (ver Figura 2.1). 


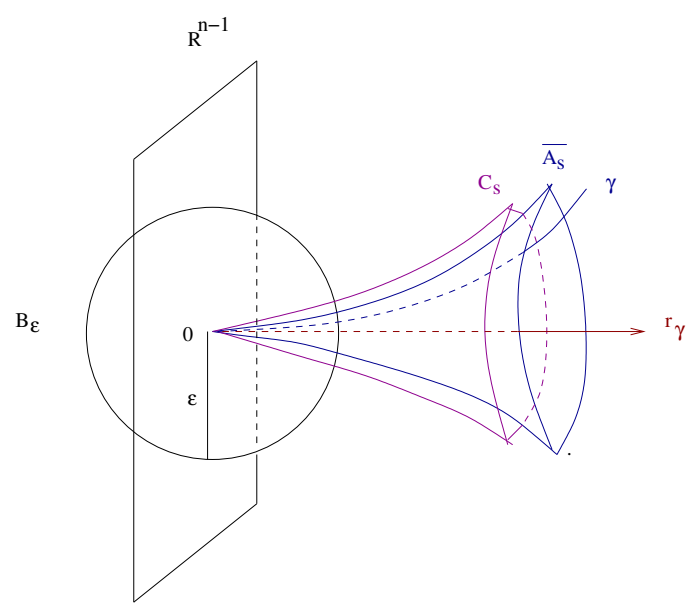

Figura 2.1: A hipótese $\left(H_{3}\right)$

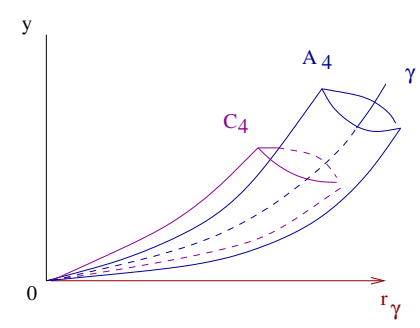

Figura 2.2: Sem a hipótese $\left(H_{3}\right)$

Notemos que sem a hipótese $\left(H_{3}\right)$ o resultado enunciado no lema acima é falso, para ver isto considere, em $\mathbb{R}^{2}, \Pi(x, y)=\left(y-x^{2}\right)\left(y-3 x^{2}\right)$ e a curva $\gamma(t)=\left(t, 2 t^{2}\right), t \geq 0$. É imediato ver que $\gamma$ é tangente em $0^{+}$ao semi-eixo $x \geq 0$ e $\Pi(\gamma(t))=-t^{4}$ enquanto $\Pi(x, 0)=3 x^{4}$ (ver Figura 2.2).

Além disso, é interessante destacar que o Lema 2.3.1 apresenta apenas conclusões sobre o sinal de $j^{s} \Pi$ em semi retas tangentes a curvas em $A_{s}$ e não em $C_{s}$, o exemplo a seguir esclarece este ponto.

Considere em $\mathbb{R}^{3}$ o polinômio $f(x, y, z)=\left(x-8 z^{2}\right)^{2}+z^{4}-y^{6}$, no semi-eixo $y \geq 0$ este polinômio satisfaz $f(0, y, 0)=-y^{6}$, isto é, este semi-eixo menos a origem está contido no conjunto $f^{-1}(-\infty, 0)$. Notemos que a derivada radial $F(x, y, z)=\langle\nabla f(x, y, z),(x, y, z)\rangle$, é um polinômio que pode ser escrito como

$$
F(x, y, z)=2\left[x-(12+\sqrt{14}) z^{2}\right]\left[x-(12-\sqrt{14}) z^{2}\right]-6 y^{6}
$$




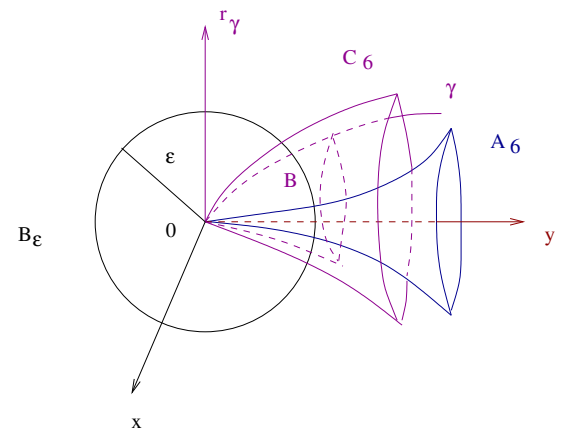

Figura 2.3: $\gamma \subset C_{6} \backslash \overline{A_{6}}$ não satisfaz a conclusão do Lema 2.3.1

notemos também que no conjunto

$B:=\left\{(x, y, z) \in \mathbb{R}^{3} \mid(12-\sqrt{14}) z^{2} \leq x \leq(12+\sqrt{14}) z^{2}, \quad-a z^{2} \leq y \leq a z^{2}\right\}$

onde $\left[(4+\sqrt{14})^{2}+1\right]^{\frac{1}{6}}<a<2$, o polinômio

$$
G(x, y, z):=2\left[x-(12+\sqrt{14}) z^{2}\right]\left[x-(12-\sqrt{14}) z^{2}\right]
$$

satisfaz $G\left((12 \pm \sqrt{14}) z^{2}, \pm a z^{2}, z\right)=0$ e como $F(x, y, z)<G(x, y, z)$ resulta que vale a inclusão $B \subset C_{6}$, onde $C_{6}$ é a componente conexa de $F^{-1}(-\infty, 0)$ que contém o semi-eixo $y \geq 0$. Por outro lado, nas curvas $\left((12 \pm \sqrt{14}) z^{2}, \pm a z^{2}, z\right)$ da fronteira de $B$ vê-se que

$$
f\left((12 \pm \sqrt{14}) z^{2}, \pm a z^{2}, z\right)=\left((4 \pm \sqrt{14})^{2}+1-a^{6} z^{8}\right) z^{4}
$$

daqui, se $\varepsilon=\frac{\left[(4-\sqrt{14})^{2}+1\right]^{\frac{1}{8}}}{a^{\frac{3}{4}}}$ temos que $f\left((12 \pm \sqrt{14}) z^{2}, \pm a z^{2}, z\right)>0$ para todo $0<z<\varepsilon$, portanto

$$
\left(\overline{A_{6}} \backslash\{0\}\right) \cap B_{\varepsilon} \subset B \cap B_{\varepsilon}
$$

onde $A_{6}$ é a componente conexa de $f^{-1}(-\infty, 0)$ que contém o semi-eixo $y \geq 0$. Mostramos, de fato, a inclusão

$$
\left(\overline{A_{6}} \backslash\{0\}\right) \cap B_{\varepsilon} \subset C_{6} \cap B_{\varepsilon} .
$$

Mas note que, a curva $\gamma(t)=\left((12+\sqrt{14}) t^{2}, a t^{2}, t\right), t \geq 0$ é tangente em $0^{+}$ao semi-eixo $z \geq 0, \gamma(0)=0$, e vale $F(\gamma(t))=-6 a^{6} t^{12}$, e se $\lambda v$ é a parametrização do semi-eixo $z \geq 0$, onde $v=(0,0,1)$ e $\lambda \geq 0$ vale também $f(\lambda v)=65 \lambda^{4}$, e $F(\lambda v)=4 f(\lambda v)$, isto é, a conclusão do Lema 2.3.1 não é válida para as curvas em $\left[C_{6} \backslash\left(\overline{A_{6}} \backslash\{0\}\right)\right] \cap B_{\varepsilon}$ (ver Figura 2.3). 


\section{Capítulo 3}

\section{Cones tangentes e $s$-resistência}

Neste capítulo será construido um cone positivo $\mathcal{K}_{s} \subset \mathbb{R}^{n}$ de vértice na origem, no qual terá sentido apresentar nosso resultado principal de $s$-resistência. Entendemos como cone de vértice na origem a um conjunto $M \subset \mathbb{R}^{n}$ tal que para cada $q \in M$ e todo $\lambda \in \mathbb{R}$ tem-se $\lambda q \in M ; M$ será chamado cone positivo se esta propriedade vale para todo $\lambda \geq 0$.

Lembremos que, a menos de menção explícita em contrário, $\Omega$ será uma vizinhança aberta da origem de $\mathbb{R}^{n}$ e $J^{s}(\Omega, \mathbb{R})$ representa o conjunto das funções de $\Omega$ em $\mathbb{R}$, de classe $C^{2}$ e que tem jato pontual de ordem $s$ em 0 .

Lembremos também que, a energia potencial $\Pi \in J^{s}(\Omega, \mathbb{R})$ satisfaz as hipóteses:

$\left(H_{1}\right) j^{\ell} \Pi(q) \geq 0$ numa vizinhança da origem $0 \in \Omega$, se $\ell \leq s-1$;

$\left(H_{2}\right) j^{s} \Pi$ mostra que $\Pi$ não tem mínimo na origem $0 \in \Omega$;

$\left(H_{3}\right)$ existe $\varepsilon>0$ com $\overline{B_{\varepsilon}} \subset \Omega$, tal que $\left(\bar{A}_{s} \backslash\{0\}\right) \cap B_{\varepsilon} \subset C_{s}^{\circ} \cap B_{\varepsilon}$.

\section{$3.1 \quad \mathrm{O}$ cone $Z_{s-1}$}

Como no capítulo anterior, se $\Pi \in J^{s}(\Omega, \mathbb{R})$ então denotemos $P_{s}(q)=$ $\left\langle\nabla j^{s} \Pi(q), q\right\rangle$ e por $A_{s}$ e $C_{s}$ os conjuntos semi-algébricos

$$
A_{s}:=\left\{q \in \Omega \mid j^{s} \Pi(q)<0\right\}, \quad C_{s}:=\left\{q \in \Omega \mid P_{s}(q)<0\right\} .
$$

Lembremos que se $\gamma:[0, \rho] \rightarrow \Omega$ é uma curva algébrica com $\gamma(0)=0$ e $\gamma(t) \neq 0$ se $0<t \leq \rho$, denota-se por $r_{\gamma}$ a semireta tangente a $\gamma$ em $0^{+}$.

Uma consequência direta do Lema 2.3.1 é o seguinte 
Corolário 3.1.1 Se $\Pi \in J^{s}(\Omega, \mathbb{R})$ satisfaz a hipótese $\left(H_{3}\right)$ e, além disso, $j^{s} \Pi$ é o primeiro jato que mostra que $\Pi$ não tem minimo na origem, então, para toda curva algébrica $\gamma:[0, \rho] \rightarrow \mathbb{R}^{n}$ tal que $\gamma(0)=0$ e $\gamma(t) \in \bar{A}_{s} \backslash\{0\}$ se $0<t \leq \rho$, tem-se que $\left.\Pi_{\ell}\right|_{r_{\gamma}} \equiv 0$ para todo $\ell \leq s-1$ e $\Pi_{s}(q)<0$ para cada $q \in r_{\gamma} \backslash\{0\}$.

DEMONSTRAÇÃO

Seja uma curva algébrica $\gamma:[0, \rho] \rightarrow \mathbb{R}^{n}$ tal que $\gamma(0)=0$ e $\gamma(t) \in \bar{A}_{s} \backslash\{0\}$ se $0<t \leq \rho$; parametrizemos a semi-reta $r_{\gamma}=\left\{\tau q_{0}\right\}$ com $\tau \geq 0$ para algum $q_{0} \in r_{\gamma} \backslash\{0\}$. Do Lema 2.3.1 segue que

$$
j^{s} \Pi(q)<0, \quad \forall q \in\left(r_{\gamma} \backslash\{0\}\right) \cap B_{\varepsilon_{0}}
$$

para algum $0<\varepsilon_{0}<1$. Usando $j^{s} \Pi=\sum_{\ell \leq s-1} \Pi_{\ell}+\Pi_{s}$ e a homogeneidade das funções $\Pi_{\ell}$, podemos escrever

$$
j^{s} \Pi\left(\tau q_{0}\right)=\sum_{\ell \leq s-1} \tau^{\ell} \Pi_{\ell}\left(q_{0}\right)+\tau^{s} \Pi_{s}\left(q_{0}\right) .
$$

Suponha, por absurdo, que para algum $\ell \leq s-1,\left.\Pi_{\ell}\right|_{r_{\gamma}} \neq 0$, tomemos $\ell_{0}=\min \left\{\ell \leq s-1:\left.\Pi_{\ell}\right|_{r_{\gamma}} \neq 0\right\}$, então $\left.\Pi_{\ell_{0}}\right|_{r_{\gamma}}$ é uma função homogênea de grau $\ell_{0}$, portanto ou $\left.\Pi_{\ell_{0}}\right|_{r_{\gamma}}$ tem mínimo estrito em 0 ou tem máximo estrito em 0. No primeiro caso $\Pi_{\ell_{0}}\left(q_{0}\right)>0$ e $j^{s} \Pi\left(\tau q_{0}\right)=\tau^{\ell_{0}} \Pi_{\ell_{0}}\left(q_{0}\right)+o\left(\left|q_{0}\right|^{s}\right)$, donde $\left.j^{s} \Pi\right|_{r_{\gamma}}$ tem mínimo estrito local em 0, contrariando o Lema 2.3.1. No segundo caso $j^{\ell_{0}} \Pi$ mostra que $\Pi$ não tem mínimo na origem, contrariando a hipótese, pois $\ell_{0}<s$.

Observação 3.1.2 Considere

$$
Z_{s-1}:=\bigcap_{\ell=k}^{s-1}\left\{q \in \Omega \mid \Pi_{\ell}(q)=0\right\} \backslash\{0\} .
$$

Observemos que, $Z_{s-1} \subset\left(j^{s-1} \Pi\right)^{-1}(\{0\})$ e, da homogeneidade das funções $\Pi_{\ell}$, segue que $Z_{s-1} \cup\{0\}$ é um cone de vértice em 0 .

Observação 3.1.3 O Corolário 3.1.1, mostra que se $\Pi \in J^{s}(\Omega, \mathbb{R})$ e $j^{s} \Pi$ é o primeiro jato que mostra que $\Pi$ não tem minimo na origem, então existe pelo menos uma semi-reta $r \subset Z_{s-1}$ de origem em 0 tal que $\Pi_{s}(q)<0$ para todo $q \in r \backslash\{0\}$. 
Proposição 3.1.4 Suponha que $\Pi$ satisfaz as hipóteses $\left(H_{1}\right)-\left(H_{3}\right)$, seja uma semi-reta $r \subset Z_{s-1}$ de origem em 0 e denotemos por $\Delta_{r}$ à componente conexa de $Z_{s-1}$ que contém $r$. Se $\Pi_{s}\left(q_{0}\right)<0$ para algum $q_{0} \in r \backslash\{0\}$, então $\Pi_{s}(q)<0$ para todo $q \in \Delta_{r} \backslash\{0\}$.

DemonstraçÃo

Da conexidade de $\Delta_{r}$, basta mostrar que $\Pi_{s}(q) \neq 0$ para todo $q \in \Delta_{r} \backslash\{0\}$. Suponha por absurdo que, para algum $q_{1} \in \Delta_{r} \backslash\{0\}$, vale $\Pi_{s}\left(q_{1}\right)=0$. Notemos primeiro que na reta $\ell=\left\{t q_{0} \mid t \in \mathbb{R}\right\}$ tem-se $\Pi_{s}\left(t q_{0}\right)=t^{s} \Pi_{s}\left(q_{0}\right)$, assim o único ponto de $\ell$ onde $\Pi_{s}$ anula-se é a origem, portanto $q_{1} \notin \ell$. Tomemos então $q^{*}$ o ponto do segmento $\overline{q_{0} q_{1}}$ mais afastado de $q_{1}$ tal que $\Pi_{s}\left(q^{*}\right)=0$ e $\Pi_{s}(q)<0$ se $q$ está no segmento $\overline{q_{0} q^{*}} \backslash\left\{q^{*}\right\}$. Então, pelas observações precedentes, $q^{*} \neq 0$, e temos que $q^{*} \in \bar{A}_{s} \backslash\{0\}$ e $j^{s} \Pi\left(q^{*}\right)=0$, contrariando $\left(H_{3}\right)$.

\subsection{Construindo o cone $\mathcal{K}_{s}$}

Se $r \subset Z_{s-1}$ é uma semi-reta de origem em 0 e $q \in r \backslash\{0\}$ um ponto fixado, podemos considerar aqui o hiperplano $[r]^{\perp}$ ortogonal a $r$ que passa por $q$. Vamos construir um cone fechado $K_{r} \subset \mathbb{R}^{n}$ contendo $r$ e de vértice na origem; simplesmente consideramos uma bola fechada $B \subset[r]^{\perp}$ de centro em $q$ e raio $\delta>0$, com $B \cap Z_{s-1} \subset \Delta_{r}$, e definindo $K_{r}$ como

$$
K_{r}:=\bigcup_{x \in B} L_{x}, \quad L_{x}:=\{\lambda x \mid 0 \leq \lambda\}
$$

Este cone fechado será útil no futuro para uma construção mais sofisticada.

Proposição 3.2.1 Suponha que $\Pi$ satisfaz as hipóteses $\left(H_{1}\right)-\left(H_{3}\right)$, seja uma semi-reta $r \subset Z_{s-1}$ de origem em 0 tal que $\Pi_{s}(q)<0$ para todo $q \in$ $r \backslash\{0\}$ e considere a componente conexa $\Delta_{r}$ de $Z_{s-1}$ que contém $r$. Então existe um cone fechado $K_{r} \subset \mathbb{R}^{n}$ de vértice na origem tal que

1. $r \backslash\{0\} \subset\left(K_{r}\right)^{\circ}$,

2. $\Pi_{s}(q)<0$ para todo $q \in K_{r} \backslash\{0\}$,

3. $K_{r} \cap Z_{s-1} \subset \Delta_{r}$. 


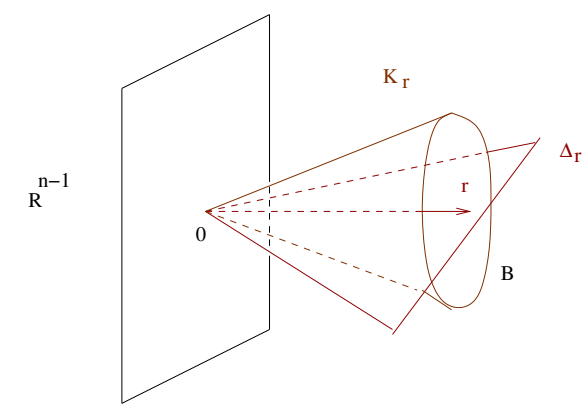

Figura 3.1: O cone $K_{r}$ e o cone $\Delta_{r} \subset Z_{s-1}$.

\section{DEMONSTRAÇÃO}

Fixemos um ponto $q_{0} \in r \backslash\{0\}$ e escolhamos um número $0<\delta_{0}<1$ pequeno o suficiente tal que $B \cap Z_{s-1} \subset \Delta_{r}$, onde $B \subset[r]^{\perp}$ é a bola fechada de centro em $q_{0}$ e raio $\delta_{0}>0$ de $[r]^{\perp}$; como feito acima, o conjunto $K_{r}$ dado por

$$
K_{r}:=\bigcup_{x \in B} L_{x}, \quad L_{x}:=\{\lambda x \mid 0 \leq \lambda\}
$$

é de fato um cone fechado de vértice em 0 e como vale $L_{q_{0}}=r$, segue então o item 1. Como $\Pi_{s}\left(q_{0}\right)<0$, da continuidade da função $\Pi_{s}$ segue que eventualmente diminuindo $\delta_{0}>0$, se necessário, tem-se $\Pi_{s}(x)<0$ para todo $x \in B$, e sendo $\Pi_{s}$ uma função homogênea tem-se que $\Pi_{s}(q)<0$ para todo $q \in L_{x} \backslash\{0\}$ e todo $x \in B$, consequentemente $\Pi_{s}(q)<0$ para todo $q \in K_{r} \backslash\{0\}$, isto mostra o item 2. Para obter o item 3 , note que, como $K_{r}$ e $Z_{s-1}$ são cones de vértice na origem, então, se $y \in K_{r} \cap Z_{s-1}, t y \in K_{r} \cap Z_{s-1}$, para todo $t>0$, assim tomando $t^{\star}=\frac{\left|q_{0}\right|}{|y|}$, tem-se $t^{\star} y \in B \cap Z_{s-1}$, portanto

$$
K_{r} \cap Z_{s-1}=\bigcup_{x \in B}\left(L_{x} \cap Z_{s-1}\right)=\bigcup_{x \in B \cap Z_{s-1}} L_{x}
$$

Como $B \cap Z_{s-1} \subset \Delta_{r}$, resulta da conexidade de $\Delta_{r}$ que $y \in \Delta_{r}$, concluindo a demonstração (ver Figura 3.1).

Observação 3.2.2 A Proposição 3.2.1 diz que, para cada semi-reta $r \subset$ $Z_{s-1}$ de origem em 0 tal que $\Pi_{s}(q)<0$ para todo $q \in r \backslash\{0\}$, o cone $K_{r}$ construido só intersepta $Z_{s-1}$ na componente conexa $\Delta_{r}$ que contém $r$. 
Corolário 3.2.3 Suponha que $\Pi$ satisfaz as hipóteses $\left(H_{1}\right)-\left(H_{3}\right)$, e considere uma semi-reta $r \subset Z_{s-1}$ de origem em 0 tal que $\Pi_{s}(q)<0$ para todo $q \in r \backslash\{0\}$, e seja $\Delta_{r}$ a componente conexa de $Z_{s-1}$ que contém $r$. Então existe um cone $\mathcal{K}_{s} \subset \mathbb{R}^{n}$ de vértice na origem tal que

(a) $\Delta_{r} \backslash\{0\} \subset\left(\mathcal{K}_{s}\right)^{\circ}$.

(b) Se $q \in \partial \mathcal{K}_{s} \backslash\{0\}$ existe $\ell \in\{k, \cdots, s-1\}$ tal que $\Pi_{\ell}(q)>0$ e $\Pi_{j}(q)=0$ para $j<\ell$.

(c) $\Pi_{s}(q)<0$ para todo $q \in \mathcal{K}_{s} \backslash\{0\}$.

\section{DEMONSTRAÇÃO}

Da Proposição 3.1.4, temos que para cada semi-reta $\tilde{r} \subset \Delta_{r}$ de origem em 0 tem-se $\Pi_{s}(q)<0$ para todo $q \in \tilde{r} \backslash\{0\}$ e da Proposição 3.2.1, existe um cone fechado $K_{\tilde{r}} \subset \mathbb{R}^{n}$ de vértice na origem com as propriedades lá descritas, isto é:

1. $r \backslash\{0\} \subset\left(K_{r}\right)^{\circ}$,

2. $\Pi_{s}(q)<0$ para todo $q \in K_{r} \backslash\{0\}$,

3. $K_{r} \cap Z_{s-1} \subset \Delta_{r}$.

Definamos o conjunto $\mathcal{K}_{s}$ como sendo

$$
\mathcal{K}_{s}:=\bigcup_{\tilde{r} \subset \Delta_{r}} K_{\tilde{r}}
$$

Seja $q \in \mathcal{K}_{s} \backslash\{0\}$ arbitrário, então $q \in K_{\tilde{r}} \backslash\{0\}$ para alguma semi-reta $\tilde{r} \subset \Delta_{r}$, tal que $\tilde{r} \backslash\{0\} \subset K_{\tilde{r}}^{\circ}$ d daqui segue que, $\Pi_{s}(q)<0$ e além disto, para cada $\lambda \geq 0$ vale $\lambda q \in K_{\tilde{r}}$; isto mostra o item $(c)$ e que $\mathcal{K}_{s}$ é de fato um cone de vértice em 0 .

Notemos que para todo $\tilde{r} \subset \Delta_{r}$, o conjunto $\bigcup_{\tilde{r} \subset \Delta_{r}}\left(K_{\tilde{r}}\right)^{\circ}$ é aberto e $\bigcup_{\tilde{r} \subset \Delta_{r}}\left(K_{\tilde{r}}\right)^{\circ} \subset \mathcal{K}_{s}$, portanto $\bigcup_{\tilde{r} \subset \Delta_{r}}\left(K_{\tilde{r}}\right)^{\circ} \subset\left(\mathcal{K}_{s}\right)^{\circ}$, e daqui obtemos as seguintes inclusões

$$
\left(\Delta_{r} \backslash\{0\}\right)=\bigcup_{\tilde{r} \subset \Delta_{r}}(\tilde{r} \backslash\{0\}) \subset \bigcup_{\tilde{r} \subset \Delta_{r}}\left(K_{\tilde{r}}\right)^{\circ} \subset\left(\mathcal{K}_{s}\right)^{\circ},
$$

o que mostra o item $(a)$.

Agora note que $\mathcal{K}_{s} \cap Z_{s-1}=\bigcup_{\tilde{r} \subset \Delta_{r}} K_{\tilde{r}} \cap Z_{s-1}$ e, pela Proposição 3.2.1 $K_{\tilde{r}} \cap Z_{s-1} \subset \Delta_{r} \backslash\{0\}$, assim

$$
\mathcal{K}_{s} \cap Z_{s-1} \subset \Delta_{r} \backslash\{0\} \subset\left(\mathcal{K}_{s}\right)^{\circ} .
$$




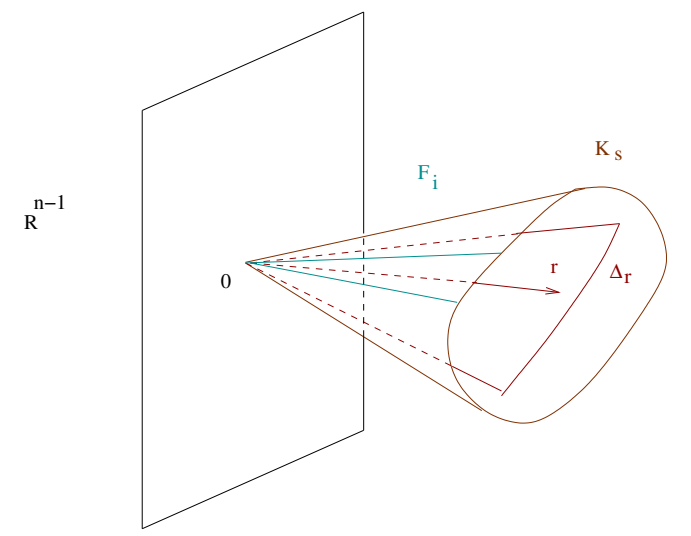

Figura 3.2: $\mathrm{O}$ cone $\mathcal{K}_{s}$ e algum cone $F_{i} \subset \partial \mathcal{K}_{s} \backslash\{0\}(k \leq i \leq s-1)$.

Como $\overline{Z_{s-1}}=Z_{s-1} \cup\{0\}$, é claro que se $q \in \overline{Z_{s-1}}$ e $q \neq 0$, então $q \in Z_{s-1}$, o que mostra que $\partial \mathcal{K}_{s} \cap Z_{s-1} \cap B_{\delta}=\emptyset$, para todo $\delta>0$.

Portanto, se $q \in \partial \mathcal{K}_{s}$ e $q \neq 0$, então $q \in\left(Z_{s-1}\right)^{c}$, e como

$$
\left(Z_{s-1}\right)^{c}=\bigcup_{\ell=k}^{s-1}\left\{q \in \Omega \mid \Pi_{\ell}(q) \neq 0\right\}
$$

vem que, para todo $q \in \partial \mathcal{K}_{s} \backslash\{0\}$, existe $\ell_{1} \in\{k, \ldots, s-1\}$ tal que $\Pi_{\ell_{1}}(q) \neq 0$.

Podemos considerar o menor destes números $\ell_{1}(q):=\min \left\{\ell_{1} \in\{k, \cdots, s-\right.$ $\left.1\} \mid \Pi_{\ell_{1}}(q) \neq 0\right\}$, e definir subconjuntos $F_{\ell} \subset \partial \mathcal{K}_{s} \backslash\{0\}$ da forma

$$
F_{\ell}:=\left\{q \in \partial \mathcal{K}_{s} \backslash\{0\} \mid \ell_{1}(q)=\ell\right\}, \quad \ell \in\{k, \ldots, s-1\} .
$$

Note que (ver Figura 3.2)

$$
\bigcup_{\ell=k}^{s-1} F_{\ell}=\left(\partial \mathcal{K}_{s} \backslash\{0\}\right) .
$$

Proposição 3.2.4 Suponha que $\Pi$ satisfaz as hipóteses $\left(H_{1}\right)-\left(H_{3}\right)$. Então existem $\varepsilon>0$ e $m>0$ tal que o cone $\mathcal{K}_{s} \subset \mathbb{R}^{n}$ dado no Corolário 3.2.3 satisfaz:

(i) $j^{s-1} \Pi(q) \geq \frac{m}{2}|q|^{s-1}, \quad \forall q \in\left(\partial \mathcal{K}_{s} \backslash\{0\}\right) \cap B_{\varepsilon}$, 
(ii) $P_{s-1}(q) \geq(s-1) \frac{m}{2}|q|^{s-1}, \quad \forall q \in\left(\partial \mathcal{K}_{s} \backslash\{0\}\right) \cap B_{\varepsilon}$.

\section{DEMONSTRAÇÃO}

Tem-se que $\partial \mathcal{K}_{s}$ é um cone de vértice na origem. Seja $S$ a esfera de centro na origem e raio 1 ; o conjunto $\partial \mathcal{K}_{s} \cap S$ é compacto.

Se $x \in \partial \mathcal{K}_{s} \cap S$ então $x \in F_{\ell}$ para algum $\ell \in\{k, \ldots, s-1\}$ e portanto $\Pi_{\ell}(x)>0$. Existe $\varepsilon_{x}>0$ tal que $\Pi_{\ell}(q)>0$ para todo $q \in\left(\partial \mathcal{K}_{s} \cap S\right) \cap \overline{B_{\varepsilon_{x}}(x)}$; podemos definir $B_{x}=\left(\partial \mathcal{K}_{s} \cap S\right) \cap \overline{B_{\varepsilon_{x}}(x)}$ e considerar

$$
m_{x}=\min _{q \in B_{x}}\left\{\Pi_{\ell}(q)\right\}>0
$$

Se $\operatorname{Cone}\left(B_{x}\right)$ é o cone de vértice na origem gerado por $B_{x}$, e $q \in \operatorname{Cone}\left(B_{x}\right)$, $q \neq 0$, então (ver Figura 3.3)

$$
j^{\ell} \Pi(q)=j^{\ell-1} \Pi(q)+\Pi_{\ell}(q) \geq \Pi_{\ell}(q)=|q|^{\ell} \Pi_{\ell}\left(\frac{q}{|q|}\right) \geq m_{x}|q|^{\ell} .
$$

Para $s>\ell+1$, escrevendo $j^{s-1} \Pi=j^{\ell} \Pi+j_{\ell}^{s-1} \Pi$, vem $j_{\ell}^{s-1} \Pi=\Pi_{\ell+1}+\ldots+$ $\Pi_{s-1}$, e portanto $j^{\ell}\left(j_{\ell}^{s-1} \Pi\right)=0$. Se $q \in \operatorname{Cone}\left(B_{x}\right), q \neq 0$, então

$$
j^{s-1} \Pi(q)=j^{\ell} \Pi(q)+j_{\ell}^{s-1} \Pi(q) \geq m_{x}|q|^{\ell}+j_{\ell}^{s-1} \Pi(q),
$$

e como $j^{\ell}\left(j_{\ell}^{s-1} \Pi\right)=0$ existe $\rho_{x}>0$ tal que se $0<|q|<\rho_{x}$ então

$$
\left|j_{\ell}^{s-1} \Pi(q)\right| \leq \frac{m_{x}}{2}|q|^{\ell} .
$$

Assim, se $0<|q|<\rho_{x}$ e $q \in \operatorname{Cone}\left(B_{x}\right)$, tem-se

$$
j^{s-1} \Pi(q) \geq \frac{m_{x}}{2}|q|^{\ell} .
$$

A família $\mathcal{C}=\left\{B_{\varepsilon_{x}}(x) \mid x \in \partial \mathcal{K}_{s} \cap S\right\}$ é uma cobertura por abertos de $\partial \mathcal{K}_{s} \cap S$. Seja $\left\{B_{\varepsilon_{x_{1}}}\left(x_{1}\right), B_{\varepsilon_{x_{2}}}\left(x_{2}\right), \ldots, B_{\varepsilon_{x_{r}}}\left(x_{r}\right)\right\}$ uma subcobertura finita de $\mathcal{C}$ tal que

$$
\partial \mathcal{K}_{s} \cap S \subset B_{\varepsilon_{x_{1}}}\left(x_{1}\right) \cup B_{\varepsilon_{x_{2}}}\left(x_{2}\right) \cup \ldots \cup B_{\varepsilon_{x_{r}}}\left(x_{r}\right) .
$$

Como $B_{x_{i}}=\left(\partial \mathcal{K}_{s} \cap S\right) \cap \overline{B_{\varepsilon_{x_{i}}}\left(x_{i}\right)}, i \in\{1, \ldots, r\}$, segue que

$$
\partial \mathcal{K}_{s} \cap S \subset B_{x_{1}} \cup B_{x_{2}} \cup \ldots \cup B_{x_{r}} .
$$

Notemos que $x_{i} \in \partial \mathcal{K}_{s} \cap S, i \in\{1, \ldots, r\}$, então pelo procedimento anterior, para cada $i \in\{1, \ldots, r\}$ existem $m_{x_{i}}>0, \rho_{x_{i}}>0$ e $\ell_{i} \in\{k, \ldots, s-1\}$ tais que, se $0<|q|<\rho_{x_{i}}$ e $q \in \operatorname{Cone}\left(B_{x_{i}}\right)$ tem-se

$$
j^{s-1} \Pi(q) \geq \frac{m_{x_{i}}}{2}|q|^{\ell_{i}} .
$$


Consideremos agora os números

$$
m:=\min \left\{m_{x_{1}}, \ldots, m_{x_{r}}\right\}>0, \quad \rho:=\min \left\{\rho_{x_{1}}, \ldots, \rho_{x_{r}}\right\}>0
$$

e seja $q \in \partial \mathcal{K}_{s}, q \neq 0$; é claro que $x_{q}=\frac{q}{|q|} \in S$ e como $\partial \mathcal{K}_{s}$ é um cone de vértice na origem $x_{q}=\frac{q}{|q|} \in \partial \mathcal{K}_{s}, \operatorname{logo} x_{q} \in \partial \mathcal{K}_{s} \cap S$, portanto $x_{q} \in B_{\varepsilon_{x_{i_{q}}}}\left(x_{i_{q}}\right)$ para algum $i_{q} \in\{1, \ldots, r\}$, consequentemente tem-se que $x_{q} \in B_{x_{i_{q}}}$; e além disso observemos que $q=|q| x_{q} \in \operatorname{Cone}\left(B_{x_{i_{q}}}\right)$. Assim, se $0<|q|<\rho$ obtemos

$$
j^{s-1} \Pi(q) \geq \frac{m_{x_{i_{q}}}}{2}|q|^{\ell_{i_{q}}} \geq \frac{m}{2}|q|^{\ell_{i_{q}}} .
$$

Portanto, se $0<\varepsilon^{\prime}<\min \{\rho, 1\}$ tem-se

$$
j^{s-1} \Pi(q) \geq \frac{m}{2}|q|^{\ell_{i q}} \geq \frac{m}{2}|q|^{s-1}, \quad \forall q \in\left(\partial \mathcal{K}_{s} \backslash\{0\}\right) \cap B_{\varepsilon^{\prime}}
$$

o qual mostra o item $(i)$ em $B_{\varepsilon^{\prime}}$.

Para ver o item $(i i)$ note que, como $j^{s-1} \Pi(q)=\sum_{\ell=k}^{s-2} \Pi_{\ell}(q)+\Pi_{s-1}(q)$, podemos escrever a seguinte estimativa

$$
\Pi_{s-1}(q) \geq \frac{m}{2}|q|^{s-1}-\sum_{\ell=k}^{s-2} \Pi_{\ell}(q), \quad \forall q \in\left(\partial \mathcal{K}_{s} \backslash\{0\}\right) \cap B_{\varepsilon^{\prime}}
$$

Pela hipótese $\left(H_{1}\right)$ vale $j^{\ell} \Pi(q) \geq 0$ se $k \leq \ell \leq s-1$ e $q \in B_{\mathcal{\varepsilon}^{\prime}}$; e como $j^{\ell} \Pi(q)=j^{\ell-1} \Pi(q)+\Pi_{\ell}(q)=\sum_{i=k}^{\ell-1} \Pi_{i}(q)+\Pi_{\ell}(q)$, podemos escrever

$$
\Pi_{\ell}(q) \geq-\sum_{i=k}^{\ell-1} \Pi_{i}(q), \quad \forall q \in B_{\varepsilon^{\prime}}, \quad k+1 \leq \ell \leq s-1
$$

Dado $q \in\left(\partial \mathcal{K}_{s} \backslash\{0\}\right) \cap B_{\varepsilon^{\prime}}$ temos que $t q \in \partial \mathcal{K}_{s} \backslash\{0\}$ para todo $t>0$. Como vale $\frac{\ell}{\ell+1}<\frac{\ell+1}{\ell+2}$ para cada $\ell \in \mathbb{N}$, temos que $\frac{k}{k+1}=\min _{k \leq \ell \leq s-1}\left\{\frac{\ell}{\ell+1}\right\}$. Consideremos $0<t<\frac{k}{k+1}$, as estimativas dadas acima e a identidade

$$
P_{s-1}(q)=\left\langle\nabla j^{s-1} \Pi(q), q\right\rangle=\sum_{\ell=k}^{s-1} \ell \Pi_{\ell}(q)
$$


para mostrar as seguintes desigualdades

$$
\begin{aligned}
P_{s-1}(t q)= & \sum_{\ell=k}^{s-2} \ell t^{\ell} \Pi_{\ell}(q)+(s-1) t^{s-1} \Pi_{s-1}(q) \\
\geq & \sum_{\ell=k}^{s-2} \ell t^{\ell} \Pi_{\ell}(q)+(s-1) t^{s-1}\left(\frac{m}{2}|q|^{s-1}-\sum_{\ell=k}^{s-2} \Pi_{\ell}(q)\right) \\
= & \sum_{\ell=k}^{s-2}\left(\ell t^{\ell}-(s-1) t^{s-1}\right) \Pi_{\ell}(q)+(s-1) t^{s-1} \frac{m}{2}|q|^{s-1} \\
= & \sum_{\ell=k}^{s-3}\left(\ell t^{\ell}-(s-1) t^{s-1}\right) \Pi_{\ell}(q)+\left((s-2) t^{s-2}-(s-1) t^{s-1}\right) \Pi_{s-2}(q) \\
& +(s-1) t^{s-1} \frac{m}{2}|q|^{s-1} \\
\geq & \sum_{\ell=k}^{s-3}\left(\ell t^{\ell}-(s-1) t^{s-1}\right) \Pi_{\ell}(q)-\left((s-2) t^{s-2}-(s-1) t^{s-1}\right) \sum_{\ell=k}^{s-3} \Pi_{\ell}(q) \\
& +(s-1) t^{s-1} \frac{m}{2}|q|^{s-1} \\
= & \sum_{\ell=k}^{s-3}\left(\ell t^{\ell}-(s-2) t^{s-2}\right) \Pi_{\ell}(q)+(s-1) t^{s-1} \frac{m}{2}|q|^{s-1} \\
\vdots & \left(k t^{k}-(k+1) t^{k+1}\right) \Pi_{k}(q)+(s-1) t^{s-1} \frac{m}{2}|q|^{s-1} \\
= & (k-(k+1) t) \Pi_{k}(t q)+(s-1) \frac{m}{2}|t q|^{s-1} \cdot \\
= &
\end{aligned}
$$

Daqui, como $\Pi_{k}(t q)=j^{k} \Pi(t q) \geq 0$ e $0<t<\frac{k}{k+1}$ resulta

$$
P_{s-1}(t q) \geq(s-1) \frac{m}{2}|t q|^{s-1}, \quad \forall q \in\left(\partial \mathcal{K}_{s} \backslash\{0\}\right) \cap B_{\varepsilon^{\prime}}, \quad 0<t<\frac{k}{k+1}
$$

Portanto, se $\varepsilon=\frac{k}{k+1} \varepsilon^{\prime}$ obtemos o afirmado

$$
P_{s-1}(q) \geq(s-1) \frac{m}{2}|q|^{s-1}, \quad \forall q \in\left(\partial \mathcal{K}_{s} \backslash\{0\}\right) \cap B_{\varepsilon} .
$$




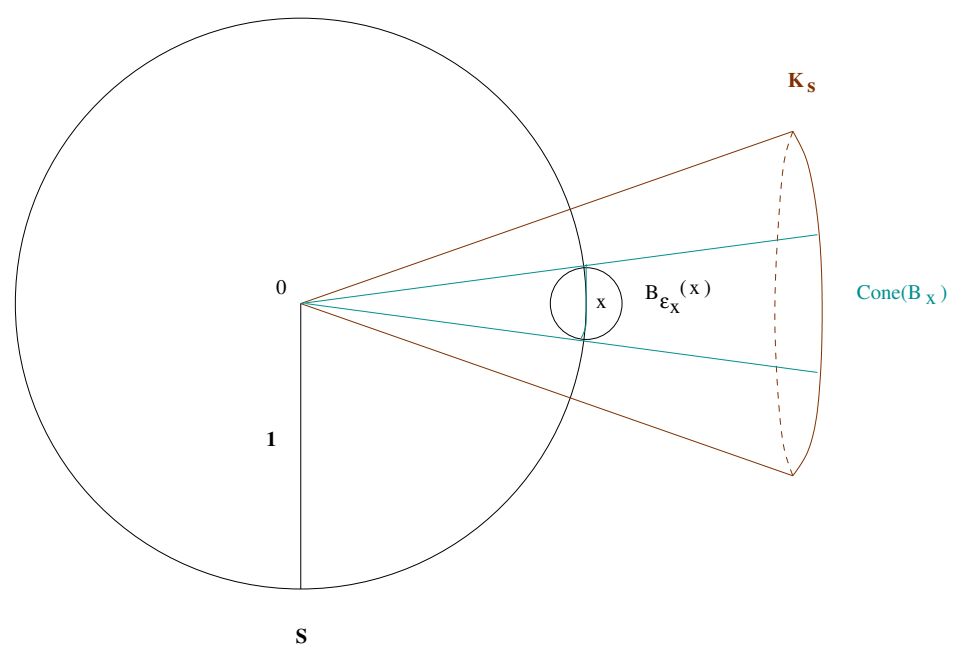

Figura 3.3: Um ponto $x \in \partial \mathcal{K}_{s} \cap S$ e o cone $\operatorname{Cone}\left(B_{x}\right)$.

Corolário 3.2.5 Suponha que $\Pi$ satisfaz as hipóteses $\left(H_{1}\right)-\left(H_{3}\right)$. Então existe $\varepsilon>0$ tal que o cone $\mathcal{K}_{s} \subset \mathbb{R}^{n}$ dado no Corolário 3.2.3, satisfaz:

$(i)^{\prime} j^{s} \Pi(q)>0$ para cada $q \in\left(\partial \mathcal{K}_{s} \backslash\{0\}\right) \cap B_{\varepsilon}$,

$(\text { ii })^{\prime} P_{s}(q)>0$ para cada $q \in\left(\partial \mathcal{K}_{s} \backslash\{0\}\right) \cap B_{\varepsilon}$.

\section{DEMONSTRAÇÃO}

Do item $(c)$ dado no Corolário 3.2.3, tem-se que $\Pi_{s}(q)<0$ para cada $q \in$ $\mathcal{K}_{s} \backslash\{0\}$; logo vale $\Pi_{s}\left(\frac{q}{|q|}\right)=|q|^{-s} \Pi_{s}(q)<0$ para cada $q \in \mathcal{K}_{s} \backslash\{0\}$, e como a esfera $\partial B_{1}$ de raio 1 é compacta, existem constantes $M_{1}>0, M_{2}>0$ tais que

$$
-M_{1} \leq \Pi_{s}\left(\frac{q}{|q|}\right) \leq-M_{2}, \quad \forall q \in\left(\mathcal{K}_{s} \backslash\{0\}\right) .
$$

Da Proposição 3.2.4, item $(i)$, existem uma constante uniforme $m>0$ e o número $0<\varepsilon^{\prime}<1$ tais que

$$
j^{s-1} \Pi(q) \geq \frac{m}{2}|q|^{s-1}, \quad \forall q \in\left(\partial \mathcal{K}_{s} \backslash\{0\}\right) \cap B_{\varepsilon^{\prime}} .
$$

Assim, para cada $q \in\left(\partial \mathcal{K}_{s} \backslash\{0\}\right) \cap B_{\varepsilon^{\prime}}$ resulta

$$
j^{s} \Pi(q)=j^{s-1} \Pi(q)+\Pi_{s}(q) \geq \frac{m}{2}|q|^{s-1}-M_{1}|q|^{s}=|q|^{s-1}\left(\frac{m}{2}-M_{1}|q|\right)
$$

e considerando $\varepsilon_{1}=\min \left\{\varepsilon^{\prime}, \frac{m}{2 M_{1}}\right\}$ obtemos o afirmado no item $(i)^{\prime}$, em $B_{\varepsilon_{1}}$. 
Analogamente, da Proposição 3.2.4, item (ii), existe $0<\varepsilon^{\prime \prime}<1$ tal que

$$
P_{s-1}(q) \geq(s-1) \frac{m}{2}|q|^{s-1}, \quad \forall q \in\left(\partial \mathcal{K}_{s} \backslash\{0\}\right) \cap B_{\varepsilon^{\prime \prime}},
$$

logo, para cada $q \in\left(\partial \mathcal{K}_{s} \backslash\{0\}\right) \cap B_{\varepsilon^{\prime \prime}}$ obtemos

$$
\begin{aligned}
P_{s}(q) & =P_{s-1}(q)+s \Pi_{s}(q) \geq(s-1) \frac{m}{2}|q|^{s-1}-s M_{1}|q|^{s} \\
& =|q|^{s-1}\left[(s-1) \frac{m}{2}-s M_{1}|q|\right],
\end{aligned}
$$

e considerando $\varepsilon_{2}=\min \left\{\varepsilon^{\prime \prime}, \frac{(s-1) m}{2 s M_{1}}\right\}$ obtemos o afirmado no item $(i i)^{\prime}$, em $B_{\varepsilon_{2}}$. Agora tome $\varepsilon=\min \left\{\varepsilon_{1}, \varepsilon_{2}\right\}$.

Observação 3.2.6 Do Corolário 3.2.3, temos que, se o conjunto

$$
M_{s-1}:=\left\{r \subset Z_{s-1}\left|\quad \Pi_{s}\right|_{(r \backslash\{0\})}<0\right\} \neq \emptyset
$$

for conexo, então $M_{s-1}=\Delta_{r}$ para alguma semi-reta $r \subset M_{s-1}$ de origem em 0 . O conjunto $M_{s-1}$ é de fato um cone positivo de vértice na origem e será chamado de cone tangente ao conjunto $\overline{A_{s}}$, pois, para qualquer curva algébrica $\gamma:[0, \rho] \longrightarrow \Omega$ com $\gamma(0)=0$ e $\gamma(t) \in \overline{A_{s}} \backslash\{0\}$ se $0<t \leq \rho$, tem-se que $r_{\gamma} \subset M_{s-1}$.

Admitindo que o cone tangente $M_{s-1}$ ao conjunto $\overline{A_{s}}$ é constituido por uma única componente conexa, temos a seguinte consequência

Corolário 3.2.7 Suponha que $\Pi$ satisfaz as hipóteses $\left(H_{1}\right)-\left(H_{3}\right)$, e seja $r$ uma semi-reta de origem 0 contida em $M_{s-1}$. Se $\tilde{A}_{s}$ e $\tilde{C}_{s}$ são, respectivamente as componentes conexas de $A_{s}$ e $C_{s}$ que contém $r \backslash\{0\}$ e $\mathcal{K}_{s}$ é o cone dado pelo Corolário 3.2.3, então existe $0<\varepsilon_{0}<1$ tal que

$$
\left(\overline{\tilde{C}_{s}} \backslash\{0\}\right) \cap B_{\varepsilon_{0}} \subset\left(\mathcal{K}_{s}\right)^{\circ} \cap B_{\varepsilon_{0}} .
$$

DEMONSTRAÇÃo

Do Lema 2.3.1, o cone tangente a $\overline{\tilde{A}_{s}} \backslash\{0\}$ é

$$
M_{s-1}=\left\{r \subset Z_{s-1}\left|\quad \Pi_{s}\right|_{(r \backslash\{0\})}<0\right\}
$$

e satisfaz a inclusão $M_{s-1} \subset \tilde{A}_{s}$ pois $\left.j^{s} \Pi\right|_{(r \backslash\{0\})}=\left.\Pi_{s}\right|_{(r \backslash\{0\})}$ para cada semireta $r \in M_{s-1}$; do item (a) dado no Corolário 3.2.3 temos $M_{s-1} \subset\left(\mathcal{K}_{s}\right)^{\circ}$, e 
do item $(b)$ segue que a fronteira $\partial \mathcal{K}_{s} \backslash\{0\}$ não é tangente a $\overline{A_{s}} \backslash\{0\}$ pois $\left(\partial \mathcal{K}_{s} \backslash\{0\}\right) \cap Z_{s-1} \cap B_{\varepsilon}=\emptyset$ para todo $\varepsilon>0$.

Além disto, do item $(i)^{\prime}$ dado no Corolário 3.2.5, existe $0<\varepsilon_{1}<1$ tal que $j^{s} \Pi(q)>0$ para cada $q \in\left(\partial \mathcal{K}_{s} \backslash\{0\}\right) \cap B_{\varepsilon_{1}}$, assim, diminuindo $\varepsilon_{1}$ se necessário, obtemos

$$
\left(\overline{\tilde{A}_{s}} \backslash\{0\}\right) \cap B_{\varepsilon_{1}} \subset\left(\mathcal{K}_{s}\right)^{\circ} \cap B_{\varepsilon_{1}}
$$

e, como do item $(c)$ dado no Corolário 3.2.3, vale $\Pi_{s}(q)<0$ para cada $q \in \mathcal{K}_{s} \backslash\{0\}$, tem-se

$$
\Pi_{s}(q)<0, \quad \forall q \in\left(\partial \tilde{A}_{s} \backslash\{0\}\right) \cap B_{\varepsilon_{1}} .
$$

Pela hipótese $\left(H_{3}\right)$ vale a inclusão $\left(\overline{\tilde{A}_{s}} \backslash\{0\}\right) \cap B_{\varepsilon_{1}} \subset \tilde{C}_{s} \cap B_{\varepsilon_{1}}$, consequentemente vale $P_{s}(q)<0$, para todo $q \in\left(\partial \tilde{A}_{s} \backslash\{0\}\right) \cap B_{\varepsilon_{1}}$, e como $P_{s}(q)=\left\langle\nabla j^{s} \Pi(q), q\right\rangle$ satisfaz a igualdade (dada na próxima seção, no Lema $3.3 .1)$

$$
P_{s}(q)=(s-1) j^{s} \Pi(q)-\sum_{\ell=k}^{s-2} j^{\ell} \Pi(q)+\Pi_{s}(q)
$$

utilizando a hipótese $\left(H_{1}\right)$ obtemos daqui que

$$
P_{s}(q) \leq \Pi_{s}(q), \quad \forall q \in\left(\partial \tilde{A}_{s} \backslash\{0\}\right) \cap B_{\varepsilon_{1}} .
$$

Lembremos que como $\mathcal{K}_{s}$ é um cone positivo de vértice na origem, fechado, existem constantes $M_{1}>0$ e $M_{2}>0$ tais que

$$
-M_{1} \leq \Pi_{s}\left(\frac{q}{|q|}\right) \leq-M_{2}, \quad \forall q \in\left(\mathcal{K}_{s} \backslash\{0\}\right) .
$$

Seja agora uma função $o\left(|q|^{s}\right)$, esta função satisfaz $\lim _{q \rightarrow 0} \frac{o\left(|q|^{s}\right)}{|q|^{s}}=0$, isto é, para $M_{2}>0$ existe $0<\tilde{\varepsilon}_{1}<\varepsilon_{1}$ tal que

$$
\left|\frac{o\left(|q|^{s}\right)}{|q|^{s}}\right|<\frac{M_{2}}{2}, \quad \forall q \in B_{\tilde{\varepsilon}_{1}} .
$$

Daqui, se $q \in\left(\mathcal{K}_{s} \backslash\{0\}\right) \cap B_{\tilde{\varepsilon}_{1}}$ tem-se

$$
\Pi_{s}(q)+o\left(|q|^{s}\right)=|q|^{s}\left[\Pi_{s}\left(\frac{q}{|q|}\right)+\frac{o\left(|q|^{s}\right)}{|q|^{s}}\right] \leq-\frac{M_{2}}{2}|q|^{s},
$$




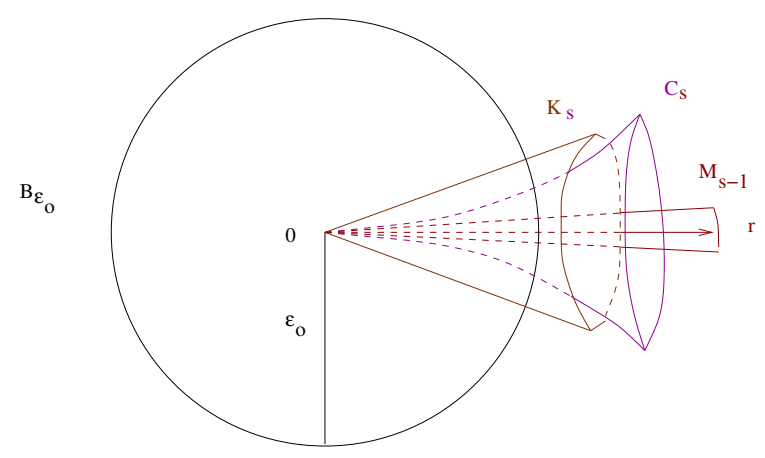

Figura 3.4: $\mathrm{O}$ conjunto $C_{s}$ e o cone $\mathcal{K}_{s}$.

consequentemente, como para cada $q \in\left(\partial \tilde{A}_{s} \backslash\{0\}\right) \cap B_{\tilde{\varepsilon}_{1}}$ existe uma semireta contida em $\left(\mathcal{K}_{s}\right)^{\circ} \cap B_{\tilde{\varepsilon}_{1}}$ passando pela origem e por $q$, vale que

$$
\Pi_{s}(q)+o\left(|q|^{s}\right) \leq-\frac{M_{2}}{2}|q|^{s}, \quad \forall q \in\left(\partial \tilde{A}_{s} \backslash\{0\}\right) \cap B_{\tilde{\varepsilon}_{1}} .
$$

Assim, usando a desigualdade

$$
P_{s}(q) \leq \Pi_{s}(q), \quad \forall q \in\left(\partial \tilde{A}_{s} \backslash\{0\}\right) \cap B_{\tilde{\varepsilon}_{1}}
$$

resulta que

$$
P_{s}(q)+o\left(|q|^{s}\right) \leq-\frac{M_{2}}{2}|q|^{s}, \quad \forall q \in\left(\partial \tilde{A}_{s} \backslash\{0\}\right) \cap B_{\tilde{\varepsilon}_{1}} .
$$

Além disto, do item $(i i)^{\prime}$ dado no Corolário 3.2.5, existe $0<\varepsilon_{2}<1$ tal que $P_{s}(q)>0$ para cada $q \in\left(\partial \mathcal{K}_{s} \backslash\{0\}\right) \cap B_{\varepsilon_{2}}$, assim, se considerarmos $\varepsilon_{0}=\min \left\{\varepsilon_{2}, \tilde{\varepsilon}_{1}\right\}$, obtemos (ver Figura 3.4)

$$
\left(\overline{\tilde{C}_{s}} \backslash\{0\}\right) \cap B_{\varepsilon_{0}} \subset\left(\mathcal{K}_{s}\right)^{\circ} \cap B_{\varepsilon_{0}} .
$$

Observação 3.2.8 A desigualdade

$$
P_{s}(q)+o\left(|q|^{s}\right) \leq-\frac{M_{2}}{2}|q|^{s}, \quad \forall q \in\left(\partial \tilde{A}_{s} \backslash\{0\}\right) \cap B_{\tilde{\varepsilon}_{1}}
$$

vista no Corolário 3.2.7 mostra uma propriedade do polinômio $P_{s}$ no conjunto $\left(\partial \tilde{A}_{s} \backslash\{0\}\right) \cap B_{\tilde{\varepsilon}_{1}}$ que na próxima seção será definida como s-resistência. 
Esta propriedade refere-se à resistência do sinal de um polinômio num conjunto. Neste caso tem-se claramente que o sinal de $P_{s}(q)+o\left(|q|^{s}\right)$ é o mesmo que o de $P_{s}(q)$ em $\left(\partial \tilde{A}_{s} \backslash\{0\}\right) \cap B_{\tilde{\varepsilon}_{1}}$, isto é, o sinal de $P_{s}(q)$ "resiste" a perturbações de ordem $o\left(|q|^{s}\right)$ no conjunto $\left(\partial \tilde{A}_{s} \backslash\{0\}\right) \cap B_{\tilde{\varepsilon}_{1}}$ em uma vizinhança da origem.

Observação 3.2.9 Se $M_{s-1}$ é constituido por mais de uma componente conexa, aplicamos a cada componente o corolário 3.2.7.

\section{$3.3 s$-resistência de $P_{s}$}

Começamos esta seção dando a seguinte estimativa para $P_{s}$

Lema 3.3.1 Se $\Pi \in J^{s}(\Omega, \mathbb{R})$, então $j^{s} \Pi(q)=\sum_{\ell=k}^{s} \Pi_{\ell}(q)$ e sua derivada radial $P_{s}(q):=\left\langle\nabla j^{s} \Pi(q), q\right\rangle$ satisfazem a seguinte igualdade:

$$
P_{s}(q)=(s-1) j^{s} \Pi(q)-\sum_{\ell=k}^{s-2} j^{\ell} \Pi(q)+\Pi_{s}(q) .
$$

Demonstração

Como $\Pi_{\ell}$ é uma função homogênea para todo $\ell \in\{k, \ldots, s\}$, obtemos do teorema de Euler que $P_{s}(q)=\sum_{\ell=k}^{s} \ell \Pi_{\ell}(q)$, e a igualdade segue diretamente.

Admitindo que os conjuntos $A_{s}$ e $C_{s}$, assim como o cone $M_{s-1}$ tangente ao conjunto $\overline{A_{s}}$, são todos constituidos por uma única componente conexa, o seguinte resultado de crucial importância é obtido:

Proposição 3.3.2 Suponha que $\Pi$ satisfaz as hipóteses $\left(H_{1}\right)-\left(H_{3}\right)$. Então existem $0<\varepsilon<1$ e um conjunto semi-algébrico $W_{s} \subset B_{\varepsilon}$ tais que $0 \in \partial W_{s}$, $e$ :

1. $\left(\bar{A}_{s} \backslash\{0\}\right) \cap B_{\varepsilon} \subset W_{s}^{\circ} \subset \bar{W}_{s} \backslash\{0\} \subset\left(C_{s}\right)^{\circ} \cap B_{\varepsilon}$.

2. $\Pi_{s}(q)<0$ para todo $q \in \bar{W}_{s} \backslash\{0\}$ e, ademais, existem constantes $\alpha>0$ e $\beta>0$ tais que:

(a) Se $q \in \partial W_{s} \backslash\{0\}$, então $j^{s} \Pi(q) \geq-\alpha \Pi_{s}(q)$;

(b) Se $q \in \bar{W}_{s} \backslash\{0\}$, então $P_{s}(q) \leq \beta \Pi_{s}(q)$. 


\section{DEMONSTRAÇÃo}

Consideremos a seguinte família a um parâmetro de conjuntos semi-algébricos $\mathcal{W}_{s}^{\lambda}:=\{q \in \Omega \mid Q(q, \lambda)<0\}$, onde $Q(q, \lambda) \in \mathbb{R}[q, \lambda]$ são polinômios definidos como

$$
Q(q, \lambda):=(s-1) j^{s} \Pi(q)+\lambda\left[-\sum_{\ell=k}^{s-2} j^{\ell} \Pi(q)+\Pi_{s}(q)\right] .
$$

Observemos que estes polinômios satisfazem o seguinte:

(i) $Q(q, 0)=(s-1) j^{s} \Pi(q)$ para todo $q \in \Omega$, o qual mostra que $\mathcal{W}_{s}^{0}=A_{s}$;

(ii) $Q(q, 1)=P_{s}(q)$ para todo $q \in \Omega$, mostrando que $\mathcal{W}_{s}^{1}=C_{s}$;

(iii) $\frac{\partial Q}{\partial \lambda}(q, \lambda)=-\sum_{\ell=k}^{s-2} j^{\ell} \Pi(q)+\Pi_{s}(q)$ para todo $q \in \Omega$, daqui que, utilizando a hipótese $\left(H_{1}\right)$, obtemos

$$
\frac{\partial Q}{\partial \lambda}(q, \lambda)<\Pi_{s}(q)<0, \quad \forall q \in \mathcal{K}_{s} \backslash\{0\}, \quad \forall \lambda \in[0,1],
$$

onde $\mathcal{K}_{s}$ é o cone construido no Corolário 3.2.7 (e que tem a estrutura dada no Corolário 3.2.3).

Consideremos $0<\varepsilon_{0}<1$ dado no Corolário 3.2.7; do item (iii), sendo as funções $Q(q, \cdot)$ decrescentes, para todo $q \in\left(\mathcal{K}_{s} \backslash\{0\}\right) \cap B_{\varepsilon_{0}}$ temos que, a família $\mathcal{W}_{s}^{\lambda}$ é crescente em $\lambda$, isto é; se $0<\lambda_{1}<\lambda_{2}<1$ valem as seguintes inclusões

$$
\left(\bar{A}_{s} \backslash\{0\}\right) \cap B_{\varepsilon_{0}} \subset \mathcal{W}_{s}^{\lambda_{1}} \cap B_{\varepsilon_{0}} \subset \mathcal{W}_{s}^{\lambda_{2}} \cap B_{\varepsilon_{0}} \subset C_{s} \cap B_{\varepsilon_{0}}
$$

Para algum número $0<\varepsilon<\varepsilon_{0}<1$, consideremos o conjunto semi-algébrico $W_{s}:=\mathcal{W}_{s}^{1 / 2} \cap B_{\varepsilon}$, é claro que $0 \in \partial W_{s}$ e que $W_{s} \subset B_{\varepsilon}$ satisfaz o item 1 . É claro também que, da inclusão

$$
\bar{W}_{s} \backslash\{0\} \subset C_{s} \cap B_{\varepsilon} \subset\left(\mathcal{K}_{s}\right)^{\circ} \cap B_{\varepsilon}
$$

e do Corolário 3.2.3, tem-se que $\Pi_{s}(q)<0$ para todo $q \in \bar{W}_{s} \backslash\{0\}$. Daqui, se $q \in \partial W_{s} \backslash\{0\}$, então $Q(q, 1 / 2)=0$, de onde obtemos a seguinte desigualdade

$$
(s-1) j^{s} \Pi(q)=-(1 / 2)\left[-\sum_{\ell=k}^{s-2} j^{\ell} \Pi(q)+\Pi_{s}(q)\right] \geq-(1 / 2) \Pi_{s}(q)
$$

o que mostra o item $2-(a) \operatorname{com} \alpha=\frac{1}{2(s-1)}$. 


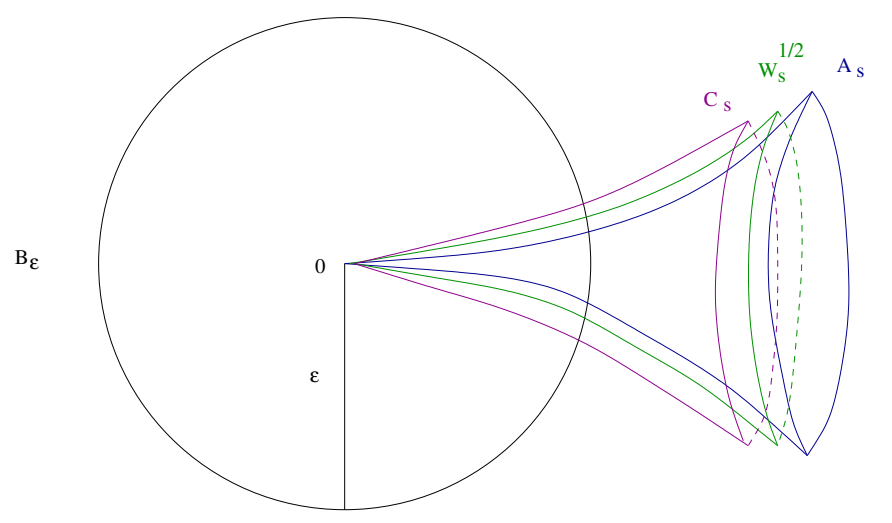

Figura 3.5: O conjunto $W_{s}$ e a $s$-resitência.

Por outro lado, se $q \in \bar{W}_{s} \backslash\{0\}$, então $Q(q, 1 / 2) \leq 0$, de onde conseguimos a segunda desigualdade

$$
\begin{aligned}
P_{s}(q) & =Q(q, 1)=Q(q, 1 / 2)+Q(q, 1)-Q(q, 1 / 2) \\
& \leq(1 / 2)\left[-\sum_{\ell=k}^{s-2} j^{\ell} \Pi(q)+\Pi_{s}(q)\right] \\
& \leq(1 / 2) \Pi_{s}(q)
\end{aligned}
$$

mostrando o item 2-(b) $\operatorname{com} \beta=\frac{1}{2}$ (ver Figura 3.5).

Consideremos a seguinte

Definição 3.3.3 Seja $U \subset \Omega$ um conjunto aberto com $0 \in \partial U$ e $f$ um polinômio de grau menor ou igual a s tal que $f(0)=0$ e $f(q)<0$ para cada $q \in U$. Dizemos que $f$ é s-resistente em $\bar{U} \backslash\{0\}$, se para toda função $h \in J^{s}(\Omega, \mathbb{R})$ com $j^{s} h \equiv 0$, existe $\varepsilon=\varepsilon(h)>0$ tal que $f(q)+h(q)<0$ para cada $q \in(\bar{U} \backslash\{0\}) \cap B_{\varepsilon}$.

Podemos agora, apresentar nosso resultado principal em termos de $s$ resistência.

Teorema 3.3.4 Se $\Pi$ satisfaz as hipóteses $\left(H_{1}\right)-\left(H_{3}\right)$, e se $\bar{W}_{s}$ é o conjunto dado na Proposição 3.3.2, então

(i) Existe $0<\varepsilon_{1}<1$ tal que $\Pi(q)>0$, para todo $q \in\left(\partial W_{s} \backslash\{0\}\right) \cap B_{\varepsilon_{1}}$. 
(ii) Existe $0<\varepsilon_{2}<1$ tal que, $P_{s}$ é s-resistente em $\left(\bar{W}_{s} \backslash\{0\}\right) \cap B_{\varepsilon_{2}}$.

\section{DEMONSTRAÇÃO}

Consideremos $0<\varepsilon_{0}<1$ obtido na Proposição 3.3.2, tal que

$$
\Pi_{s}(q)<0, \quad \forall q \in \bar{W}_{s} \backslash\{0\} \subset C_{s} \cap B_{\varepsilon_{0}} \subset\left(\mathcal{K}_{s}\right)^{\circ} \cap B_{\varepsilon_{0}} .
$$

Como em qualquer curva algébrica $\gamma:[0,1) \rightarrow \bar{W}_{s} \operatorname{com} \gamma(0)=0, \gamma(t) \neq 0$ se $t>0$, tem-se que 0 é ponto de máximo de $\left.\Pi_{s}\right|_{\gamma}$, existe $0<\varepsilon_{1}=\varepsilon_{1}\left(\varepsilon_{0}\right)<\varepsilon_{0}$ tal que

$$
\Pi_{s}(q) \leq-\varepsilon_{0}|q|^{s}, \quad \forall q \in \bar{W}_{s} \backslash\{0\} \cap B_{\varepsilon_{1}} .
$$

Na vizinhança $B_{\varepsilon_{0}}$, a função $\Pi$ pode ser escrita, por hipótese, da seguinte forma

$$
\Pi(q)=j^{s} \Pi(q)+o\left(|q|^{s}\right), \quad q \in B_{\varepsilon_{0}},
$$

onde $o\left(|q|^{s}\right)$ é uma função contínua satisfazendo $\lim _{q \rightarrow 0} \frac{o\left(|q|^{s}\right)}{|q|^{s}}=0$, portanto

$$
\left|o\left(|q|^{s}\right)\right|<\frac{\alpha \varepsilon_{0}}{2}|q|^{s}, \quad \forall q \in B_{\varepsilon_{1}} \backslash\{0\} .
$$

Assim, do item 2-(a) dado na Proposição 3.3.2, podemos obter, para todo $q \in\left(\partial W_{s} \backslash\{0\}\right) \cap B_{\varepsilon_{1}}$ a seguinte desigualdade

$$
\Pi(q)=j^{s} \Pi(q)+o\left(|q|^{s}\right) \geq-\alpha \Pi_{s}(q)+o\left(|q|^{s}\right)>\frac{\alpha \varepsilon_{0}}{2}|q|^{s},
$$

o que mostra o item $(i)$, para $\varepsilon_{1} \leq \frac{\alpha \varepsilon_{0}}{2}$.

Considere agora $0<\varepsilon_{1}<1$, obtido na Proposição 3.3.2. Sabemos que

$$
\Pi_{s}(q)<0, \quad \forall q \in \bar{W}_{s} \backslash\{0\} \subset C_{s} \cap B_{\varepsilon_{1}} \subset\left(\mathcal{K}_{s}\right)^{\circ} \cap B_{\varepsilon_{1}}
$$

e, como na prova do item anterior, existe $0<\varepsilon_{2}=\varepsilon_{2}\left(\varepsilon_{1}\right)<\varepsilon_{1}$ tal que

$$
\Pi_{s}(q) \leq-\varepsilon_{1}|q|^{s}, \quad \forall q \in \bar{W}_{s} \backslash\{0\} \cap B_{\varepsilon_{2}} .
$$

Seja agora $h \in J^{s}(\Omega, \mathbb{R})$ uma função definida em $B_{\varepsilon_{1}} \subset \Omega$, tal que $j^{s} h \equiv 0$; por definição $h(q)=o\left(|q|^{s}\right), q \in B_{\varepsilon_{1}}$, portanto,

$$
|h(q)|<\frac{\beta \varepsilon_{1}}{2}|q|^{s}, \quad \forall q \in B_{\varepsilon_{2}} \backslash\{0\} .
$$


Assim, do item 2-(b) dado na Proposição 3.3.2, podemos obter, para todo $q \in\left(\bar{W}_{s} \backslash\{0\}\right) \cap B_{\varepsilon_{2}}$ a seguinte desigualdade

$$
P_{s}(q)+h(q) \leq \beta \Pi_{s}(q)+h(q) \leq-\frac{\beta \varepsilon_{1}}{2}|q|^{s},
$$

mostrando o item (ii) para $\varepsilon_{2} \leq \frac{\beta \varepsilon_{1}}{2}$, isto é, mostra-se a $s$-resistência de $P_{s}(q):=\left\langle\nabla j^{s} \Pi(q), q\right\rangle$ no conjunto $\left(\bar{W}_{s} \backslash\{0\}\right) \cap B_{\varepsilon_{2}}$.

Observação 3.3.5 Considere a componente conexa $\Theta$ do conjunto $B_{\varepsilon_{2}} \cap$ $\Pi^{-1}((-\infty, 0))$ que contém o cone tangente $\left(M_{s-1} \backslash\{0\}\right) \cap B_{\varepsilon_{2}}$. Obtemos da Proposição 3.3 .2 que

$$
\Theta \cap\left(\partial W_{s} \backslash\{0\}\right) \cap B_{\varepsilon_{2}}=\emptyset
$$

e, como $\left(M_{s-1} \backslash\{0\}\right) \cap B_{\varepsilon_{2}} \subset \Theta \cap W_{s} \cap B_{\varepsilon_{2}} \neq \emptyset$, da conexidade de $\Theta$, obtemos a inclusão

$$
\Theta \subset W_{s} \cap B_{\varepsilon_{2}} \subset\left(\bar{W}_{s} \backslash\{0\}\right) \cap B_{\varepsilon_{2}} .
$$

Portanto, $P_{s}$ é s-resistente em $\Theta$.

Corolário 3.3.6 Suponha que $\Pi=j^{s} \Pi+R$ satisfaz as hipóteses $\left(H_{1}\right)-$ $\left(H_{3}\right)$, e que exista a função $\nabla R(q)=o\left(|q|^{s-1}\right)$. Se $\bar{W}_{s}$ é um conjunto dado na Proposição 3.3.2, então existe $0<\varepsilon_{3}<1$, tal que $\langle\nabla \Pi(q), q\rangle<0$ para todo $q \in\left(\bar{W}_{s} \backslash\{0\}\right) \cap B_{\varepsilon_{3}}$.

DemonstraÇÃo

Considerando a função $h(q):=\langle\nabla R(q), q\rangle$, temos

$$
\langle\nabla \Pi(q), q\rangle=P_{s}(q)+h(q)
$$

e o resultado segue da $s$-resistência da função $P_{s}$ dada no Teorema 3.3.4. 


\section{Capítulo 4}

\section{Aplicações da $s$-resistência de $P_{s}$}

Neste capítulo usaremos nosso resultado sobre $s$-resistência do capítulo anterior para obter uma resposta positiva à conjectura de Liapunov apresentada no Capítulo 1 para energias potenciais $\Pi \in J^{s}(\Omega, \mathbb{R})$ que satisfazem as hipóteses $\left(H_{1}\right)-\left(H_{3}\right)$. Variantes das hipóteses $\left(H_{1}\right)-\left(H_{3}\right)$ serão apresentadas e veremos em que medida a conjectura de Liapunov é ainda válida.

\subsection{Instabilidade do equilíbrio segundo Liapunov}

Estudemos aqui a estabilidade segundo Liapunov do equilíbrio $\left(q_{0}, p_{0}\right)=$ $(0,0) \in \mathbb{R}^{n} \times \mathbb{R}^{n}$, com $n \geq 2$ graus de liberdade, cuja dinâmica é governada pelo sistema hamiltoniano

$$
X_{H}(q, p)\left\{\begin{array}{l}
\dot{q}=\frac{\partial H}{\partial p}(q, p) \\
\dot{p}=-\frac{\partial H}{\partial q}(q, p)
\end{array}\right.
$$

com hamiltoniana $H(q, p)=T(q, p)+\Pi(q),(q, p) \in \Omega \times \mathbb{R}^{n}$ onde $\Omega \subset \mathbb{R}^{n}$ é uma vizinhança aberta da origem. Suporemos que a energia cinética é da forma $T(q, p):=\frac{1}{2}\langle p, B(q) p\rangle$, sendo $B(q)$ uma matriz simétrica definida positiva, de clase $C^{2}\left(\Omega, \mathbb{R}^{n^{2}}\right)$.

\subsubsection{Hipóteses $\left(H_{1}\right)-\left(H_{3}\right)$}

No que segue, dizer que o sistema hamiltoniano $X_{H}$ satisfaz as hipóteses $\left(H_{1}\right)-\left(H_{3}\right)$ será equivalente a dizer que a energia potencial $\Pi \in J^{s}(\Omega, \mathbb{R})$ 
satisfaz as hipóteses $\left(H_{1}\right)-\left(H_{3}\right)$. Admitiremos também que, se $\Pi(q)=$ $j^{s} \Pi(q)+R(q)$, então $\nabla R(q)$ é de ordem $o\left(|q|^{s-1}\right)$ em uma vizinhança da origem.

Teorema 4.1.1 Se o sistema hamiltoniano $X_{H}(q, p)$ satisfaz as hipóteses $\left(H_{1}\right)-\left(H_{3}\right)$, então $\Pi$ é uma energia potencial do tipo Četaev e, portanto, o equilíbrio $(q, p)=(0,0)$ deste sistema é instável segundo Liapunov.

\section{DEMONSTRAÇÃO}

Seja $\varepsilon>0$, o número dado no item (ii) do Teorema 3.3.4, tal que $\bar{B}_{\varepsilon} \subset \Omega$. Temos deste resultado, a existência do conjunto $W_{s}:=\mathcal{W}_{s}^{1 / 2} \cap B_{\varepsilon} \neq \emptyset \mathrm{com}$ $0 \in \partial W_{s}$ tal que $P_{s}(q):=\left\langle\nabla j^{s} \Pi(q), q\right\rangle$ é $s$-resistente em $\left(\bar{W}_{s} \backslash\{0\}\right) \cap B_{\varepsilon}$; além disso, na prova do Teorema 3.3.4 foi mostrado que se $\Theta$ é a componente conexa de $\Pi^{-1}((-\infty, 0)) \cap B_{\varepsilon}$ que contém o cone tangente $M_{s-1} \cap B_{\varepsilon}$, vale a inclusão

$$
\Theta \subset W_{s} \cap \Pi^{-1}((-\infty, 0)) \cap B_{\varepsilon} \subset\left(\bar{W}_{s} \backslash\{0\}\right) \cap B_{\varepsilon},
$$

diminuindo $\varepsilon$ se necessário. Portanto, do Corolário 3.3.6, resulta

$$
\langle\nabla \Pi(q), q\rangle<0, \quad \forall q \in \Theta .
$$

Agora vai-se apresentar, nas seções 4.1.2 e 4.1.3, casos particulares do teorema 4.1.1. De um ponto de vista formal não haveria necessidade de fazer a demonstração da instabilidade da origem nestes casos, visto serem casos particulares do nosso teorema principal, mas optamos por apresentar demonstrações explícitas para cada caso pois, além de serem mais simples do que a do Teorema 4.1.1, ilustram técnicas e procedimentos usuais nesta área, podendo eventualmente vir a ser usadas em outras situações no futuro.

\subsubsection{Hipóteses $\left(H_{1}^{1}\right)-\left(H_{3}\right)$}

Aqui substituimos nossa hipótese $\left(H_{1}\right)$ pela seguinte hipótese $\left(H_{1}^{1}\right) \Pi_{\ell} \geq 0$ numa vizinhança da origem $0 \in \Omega$, se $\ell \leq s-1$.

Corolário 4.1.2 Se o sistema hamiltoniano $X_{H}(q, p)$ satisfaz as hipóteses $\left(H_{1}^{1}\right)-\left(H_{3}\right)$, então o equilíbrio $(q, p)=(0,0)$ deste sistema é instável segundo Liapunov. 


\section{DEMONSTRAÇÃO}

É claro que a hipótese $\left(H_{1}^{1}\right)$ implica na hipótese $\left(H_{1}\right)$, então com as hipóteses $\left(H_{1}^{1}\right)-\left(H_{3}\right)$, podemos obter também um número $\varepsilon>0$ e um conjunto $W_{s}:=\mathcal{W}_{s}^{1 / 2} \cap B_{\varepsilon}$ de maneira similar ao feito na Proposição 3.3.2. Neste caso particular o conjunto $\mathcal{W}_{s}^{1 / 2}$ é obtido como um elemento da família a um parâmetro $\mathcal{W}_{s}^{\lambda}=\{q \in \Omega \mid Q(q, \lambda)<0\}$ de conjuntos semi-algébricos, onde

$Q(q, \lambda):=(s-1) j^{s} \Pi(q)+\lambda\left[\sum_{\ell=k}^{s-2}(\ell-(s-1)) \Pi_{\ell}(q)+\Pi_{s}(q)\right], \quad \lambda \in[0,1]$.

Para $\varepsilon>0$ pequeno suficiente, o conjunto $W_{s}:=\mathcal{W}_{s}^{1 / 2} \cap B_{\varepsilon}$, de fato, satisfaz a Proposição 3.3.2 com constantes $\alpha=\frac{1}{2(s-1)}$ e $\beta=\frac{1}{2}$.

\subsubsection{Hipóteses $\left(H_{1}^{2}\right)-\left(H_{3}\right)$}

Aqui substituimos nossa hipótese $\left(H_{1}\right)$ pela seguinte hipótese

$\left(H_{1}^{2}\right)$ Existe $\nu \in\{k, \ldots, s-1\}$ tal que numa vizinhança da origem $\Pi_{r} \geq 0 \mathrm{e}$ $\Pi_{\ell} \leq 0$, se $k \leq r \leq \nu$ e $\nu+1 \leq \ell \leq s-1$ e, além disso $j^{s-1} \Pi \geq 0$.

Corolário 4.1.3 Se o sistema hamiltoniano $X_{H}(q, p)$ satisfaz as hipóteses $\left(H_{1}^{2}\right)-\left(H_{3}\right)$, então o equilíbrio $(q, p)=(0,0)$ deste sistema é instável segundo Liapunov.

DEMONSTRAÇÃO

É claro que as hipóteses $\left(H_{1}^{2}\right)-\left(H_{3}\right)$ implicam nas hipóteses $\left(H_{1}\right)-\left(H_{3}\right)$, então de maneira similar ao feito na Proposição 3.3.2 conseguimos aqui também um número $\varepsilon>0$ e um conjunto $W_{s}:=\mathcal{W}_{s}^{1 / 2} \cap B_{\varepsilon}$, onde $\mathcal{W}_{s}^{1 / 2}$ neste caso particular, é obtido como um elemento da família a um parâmetro $\mathcal{W}_{s}^{\lambda}=\{q \in \Omega \mid Q(q, \lambda)<0\} \operatorname{com} \lambda \in[0,1]$, de conjuntos semi-algébricos, onde

$Q(q, \lambda)=\nu j^{s} \Pi(q)+\lambda\left[\sum_{r=k}^{\nu-1}(r-\nu) \Pi_{r}(q)+\sum_{\ell=\nu+1}^{s-1}(\ell-\nu) \Pi_{\ell}(q)+(s-\nu) \Pi_{s}(q)\right]$

Para $\varepsilon$ pequeno suficiente, o conjunto $W_{s}:=\mathcal{W}_{s}^{1 / 2} \cap B_{\varepsilon}$ também satisfaz a Proposição 3.3.2 com constantes $\alpha=\frac{s-\nu}{2 \nu}$ e $\beta=\frac{s-\nu}{2}$. 


\subsection{Análise da hipótese $\left(H_{1}\right)$}

Nesta seção, analisamos o que acontece se substituimos nossa hipótese $\left(H_{1}\right)$ pela seguinte hipótese

$\left(H_{1}^{3}\right) j^{s-1} \Pi \geq 0$ numa vizinhança da origem $0 \in \Omega$.

Ou seja, supomos que $j^{k} \Pi$ é o primeiro jato não nulo de $\Pi$ e é semidefinido positivo, $j^{s} \Pi$ é o primeiro jato de $\Pi$ que mostra que $\Pi$ não tem mínimo na origem e $j^{s-1} \Pi$ é semi-definido positivo.

Estas hipóteses pareciam, quando começamos o trabalho, "hipóteses mais naturais" para, supondo-se que $j^{s} \Pi$ é um potencial de tipo Četaev podemos concluir que $\Pi$ também seria um potencial de tipo Četaev.

Veremos que isto não é verdade, mostraremos um exemplo no plano em que $k=6, s=12, j^{12} \Pi$ mostra que $\Pi$ não tem mínimo na origem, $j^{11} \Pi$ é semidefinido positivo na origem, a derivada radial de $j^{12} \Pi$ é negativa numa componente conexa de $\left(j^{12} \Pi\right)^{-1}((-\infty, 0))$ e, apesar disso, não é verdade que a derivada radial de $\Pi$ seja negativa em alguma componente conexa de $\Pi^{-1}((-\infty, 0))$.

Isto mostra que as hipóteses consideradas na seção anterior não podem ser "muito enfraquecidas" se quisermos usar a derivada radial de $\Pi$ como função de Četaev.

Exemplo 4.2.1 Consideremos o polinômio de duas variáveis

$$
f(x, y)=\frac{8}{3} y^{6}-3 y^{4} x^{4}+\frac{9}{10} y^{2} x^{8}-\frac{1}{12} x^{12}-y^{12}
$$

na nossa notação usual $f=f_{6}+\ldots+f_{12}$, com as seguintes parcelas homogêneas

$$
\begin{aligned}
f_{6}(x, y) & =\frac{8}{3} y^{6} \geq 0, \quad f_{7}(x, y)=0 \\
f_{8}(x, y) & =-3 y^{4} x^{4} \leq 0, \quad f_{9}(x, y)=0 \\
f_{10}(x, y) & =\frac{9}{10} y^{2} x^{8} \geq 0, \quad f_{11}(x, y)=0 \\
f_{12}(x, y) & =-\frac{1}{12} x^{12}-y^{12} \leq 0 .
\end{aligned}
$$

Claro que $j^{12} f$ tem sela na origem e $j^{6} f$, seu primeiro jato não nulo, é semidefinido positivo. Note também que $j^{12} f$ mostra que $f$ não tem mínimo na origem, pois $j^{12} f(x, 0)=-\frac{x^{12}}{12}$. 
Além disso, o jato

$$
j^{11} f(x, y)=\frac{8}{3} y^{6}-3 y^{4} x^{4}+\frac{9}{10} y^{2} x^{8}=y^{2}\left(\frac{8}{3} y^{4}-3 y^{2} x^{4}+\frac{9}{10} x^{8}\right)
$$

é semi-definido positivo e só se anula no eixo $x$. Para ver isto, basta observar o polinômio

$$
g(x, y)=\left(\frac{8}{3} y^{4}-3 y^{2} x^{4}+\frac{9}{10} x^{8}\right)
$$

como um polinômio na variável $y^{2}$ e ver que o discriminante satisfaz

$$
\Delta(x)=\left(-3 x^{4}\right)^{2}-4\left(\frac{8}{3}\right)\left(\frac{9}{10} x^{8}\right)=9 x^{8}\left(1-\frac{16}{15}\right)<0, \quad \forall x \in \mathbb{R} \backslash\{0\}
$$

que juntamente com $g(x, 0)=\frac{9}{10} x^{8}>0$, mostra que $g(x, y)$ é uma função positiva que só se anula na origem.

Já o jato

$j^{8} f(x, y)=\frac{8}{3} y^{6}-3 y^{4} x^{4}=\frac{8}{3} y^{4}\left(y^{2}-\frac{9}{8} x^{4}\right)=\frac{8}{3} y^{4}\left(y-\frac{3}{2 \sqrt{2}} x^{2}\right)\left(y+\frac{3}{2 \sqrt{2}} x^{2}\right)$

não é semi-definido positivo e tem uma sela na origem.

Isso mostra que o fato do primeiro jato não nulo de $f$ ser semi-definido positivo, $j^{s} f$ ser o primeiro jato que mostra que $f$ não tem mínimo na origem e $j^{s-1} f$ ser semi-definido positivo, não é suficiente para garantir que $j^{\ell} f \geq 0$ se $\ell \leq s-1$.

Consideraremos agora a derivada radial de $f$,

$$
F(x, y)=\langle\nabla f(x, y),(x, y)\rangle=16 y^{6}-24 y^{4} x^{4}+9 y^{2} x^{8}-x^{12}-12 y^{12},
$$

e mostraremos que numa vizinhança da origem,

$$
f^{-1}((-\infty, 0)) \subset F^{-1}((-\infty, 0)) .
$$

Pode-se escrever $F(x, y)$ da seguinte forma

$$
\begin{aligned}
F(x, y) & =\left(16 y^{6}-8 y^{4} x^{4}+y^{2} x^{8}\right)+\left(-16 y^{4} x^{4}+8 y^{2} x^{8}-x^{12}\right)-12 y^{12} \\
& =\left(y-x^{2}\right)\left(y+x^{2}\right)\left(2 y-x^{2}\right)^{2}\left(2 y+x^{2}\right)^{2}-12 y^{12} .
\end{aligned}
$$

Notemos que o conjunto $L:=\left\{(x, y) \in \mathbb{R}^{2} \mid-x^{2} \leq y \leq x^{2}\right\}$ e o polinômio $Q(x, y):=\left(y-x^{2}\right)\left(y+x^{2}\right)\left(2 y-x^{2}\right)^{2}\left(2 y+x^{2}\right)^{2}$ satisfazem

$$
\begin{aligned}
Q(x, y) & \leq 0, \quad \forall(x, y) \in L, \\
Q\left(x, \pm x^{2}\right) & =0, \quad Q\left(x, \pm \frac{1}{2} x^{2}\right)=0 .
\end{aligned}
$$


Note que as curvas $\left(x, \pm x^{2}\right)$ são a fronteira de $L$ e as curvas $\left(x, \pm \frac{1}{2} x^{2}\right)$ estão no interior de $L$.

Como $F(x, y)=Q(x, y)-12 y^{12} \leq Q(x, y)$ temos que vale a inclusão

$$
L \subset C_{12} \text {, }
$$

onde $C_{12}$ é a componente conexa de $F^{-1}((-\infty, 0))$ que contém o eixo $x$.

Consideremos também $A_{12}$, a componente conexa de $f^{-1}((-\infty, 0))$ que contém o eixo $x$.

Lema 4.2.2 Existe $\varepsilon_{0}>0$ tal que $\left(\overline{A_{12}} \backslash\{0\}\right) \cap B_{\varepsilon_{0}} \subset C_{12} \cap B_{\varepsilon_{0}}$.

DEMONSTRAÇÃO

Observemos que nas curvas $\left(x, \pm x^{2}\right)$ vale a igualdade

$$
f\left(x, \pm x^{2}\right)=\left(\frac{29}{60}-x^{12}\right) x^{12} .
$$

Daqui vem que, se $\varepsilon_{0}=\left(\frac{29}{60}\right)^{\frac{1}{12}}$, então $f\left(x, \pm x^{2}\right)>0$, para $0<x<\varepsilon_{0}$. Portanto

$$
\left(\overline{A_{12}} \backslash\{0\}\right) \cap B_{\varepsilon_{0}} \subset L \cap B_{\varepsilon_{0}},
$$

e como $L \subset C_{12}$, temos que $\left(\overline{A_{12}} \backslash\{0\}\right) \cap B_{\varepsilon_{0}} \subset C_{12} \cap B_{\varepsilon_{0}}$.

Agora será exbido um conjunto aderente à origem de $\mathbb{R}^{2}$ e neste conjunto analisamos a 12-resistência dos polinômios $f$ e $F$.

Lema 4.2.3 Se $\Pi: \mathbb{R}^{2} \rightarrow \mathbb{R}$ é uma função que na origem $(x, y)=(0,0)$ tem jato 12 igual a $f$, então se $R=\left\{(x, y) \in \mathbb{R}^{2} \mid-\frac{3}{4} x^{2} \leq y \leq \frac{3}{4} x^{2}\right\}$, vale que:

(i) Existe $\varepsilon_{1}>0$ tal que $\Pi(x, y)<0$ se $(x, y) \in\left(R \cap B_{\varepsilon_{1}}\right) \backslash\{(0,0)\}$,

(ii) F não é 12-resistente em $R$.

DEMONSTRAÇÃO

Nas curvas $\left(x, \lambda x^{2}\right)$, com $\lambda \in \mathbb{R} \backslash\{0\}$, temos que $f$ satisfaz

$$
f\left(x, \lambda x^{2}\right)=h(\lambda) x^{12}-\left(\lambda x^{2}\right)^{12} \leq h(\lambda) x^{12},
$$

onde $h(\lambda)=\frac{8}{3} \lambda^{6}-3 \lambda^{4}+\frac{9}{10} \lambda^{2}-\frac{1}{12}$ é uma função par que se anula em algum ponto $\lambda_{0} \in\left(\frac{5}{6}, \frac{7}{8}\right)$ e

$$
h(\lambda)<0, \quad \forall \lambda \in\left(-\lambda_{0}, \lambda_{0}\right)
$$


além disto, em $\lambda_{1}=\sqrt{\frac{3}{8}\left(1-\frac{1}{\sqrt{5}}\right)}<\frac{3}{4},\left.h\right|_{\left(-\lambda_{0}, \lambda_{0}\right)}$ tem um máximo global, portanto

$$
f\left(x, \lambda x^{2}\right) \leq h\left(\lambda_{1}\right) x^{12}, \quad \forall \lambda \in\left[-\frac{3}{4}, \frac{3}{4}\right] .
$$

A função $\Pi$ é da forma $\Pi(x, y)=f(x, y)+k(x, y)$, e $k(x, y)$ é $o\left(|(x, y)|^{12}\right)$ na origem, então dado $\delta>0$ existe $\varepsilon_{1}=\varepsilon_{1}(\delta)>0$ satisfazendo

$$
|k(x, y)|<\delta|(x, y)|^{12}, \quad \forall(x, y) \in B_{\varepsilon_{1}}
$$

para $\delta=-\frac{h\left(\lambda_{1}\right)}{2^{7}}>0$, temos que

$\Pi(x, y) \leq h\left(\lambda_{1}\right) x^{12}-\frac{h\left(\lambda_{1}\right)}{2} x^{12}=\frac{h\left(\lambda_{1}\right)}{2} x^{12}, \quad \forall(x, y) \in\left(R \cap B_{\varepsilon_{1}}\right) \backslash\{(0,0)\}$,

isto mostra o item $(i)$.

Para ver o item (ii), basta considerar a função $F(x, y)+14 x^{14}$; podemos ver que as curvas $\left(x, \pm \frac{1}{2} x^{2}\right)$ estão no interior do conjunto $R \mathrm{e}$

$$
\begin{aligned}
F\left(x, \pm \frac{1}{2} x^{2}\right)+14 x^{14} & =Q\left(x, \pm \frac{1}{2} x^{2}\right)-12\left( \pm \frac{1}{2} x^{2}\right)^{12}+14 x^{14} \\
& =-\frac{12}{2^{12}} x^{24}+14 x^{14}
\end{aligned}
$$

tem mínimo em $x=0$. Como $F(x, y)$ nas curvas $\left(x, \pm x^{2}\right)$ tem máximo em $x=0$, isto mostra que $F(x, y)$ não é 12-resistente em $R$.

Observemos que $F(x, y)+14 x^{14}$ é a derivada radial de $f(x, y)+x^{14}$ e que a função $\Pi(x, y)=f(x, y)+x^{14}$ ainda satisfaz

$$
\Pi(x, y) \leq h\left(\lambda_{1}\right) x^{12}+x^{14}<0, \quad \forall(x, y) \in\left(R \cap B_{\varepsilon_{1}}\right) \backslash\{(0,0)\} .
$$

Isso mostra que, se $\Pi(x, y)=f(x, y)+x^{14}$, não é verdade que vale $\langle\nabla \Pi(x, y),(x, y)\rangle<0$ se $\Pi(x, y)<0$, embora $\Pi$ satisfaça $\left(H_{1}^{3}\right)-\left(H_{3}\right)$.

Com este exemplo fica claro que as hipóteses $\left(H_{1}^{3}\right)-\left(H_{3}\right)$ não são suficientes para mostrar instabilidade do equilíbrio $\left(q_{0}, 0\right)=(0,0)$ do nosso sistema hamiltoniano $X_{H}$, usando $\langle\nabla \Pi(x, y),(x, y)\rangle$ como função de Četaev. 


\subsection{Existência de trajetórias assintóticas}

Nesta seção consideramos o sistema hamiltoniano $X_{H}(q, p)$ dado na seção 4.1 satisfazendo as hipóteses $\left(H_{1}\right)-\left(H_{3}\right)$ e estudamos a existência de trajetórias deste sistema que são assintóticas à origem. Este estudo, de fato, é um problema relevante em sistemas hamiltonianos, ver por exemplo $[\mathrm{P}]$.

Para mostrar a existência de trajetórias assintóticas no nosso caso estabeleceremos um resultado que relaciona trajetórias assintóticas e funções auxiliares.

Considere $\Omega \subset \mathbb{R}^{n}$ um conjunto aberto, $f: \Omega \rightarrow \mathbb{R}^{n}$ uma função de classe $C^{1}$ e a equação diferencial

$$
\dot{x}=f(x) .
$$

Suponha que $\bar{x} \in \Omega$ e $f(\bar{x})=0$; então $\bar{x}$ é um ponto de equilíbrio de (4.1) e exibiremos uma condição suficiente para a existência de uma solução $x(t)$ de (4.1) tal que $\lim _{t \rightarrow-\infty} x(t)=\bar{x}$, que é uma solução assintótica a $\bar{x}$ para $t \rightarrow-\infty$.

É um resultado bem conhecido de Četaev que, se existe um subconjunto aberto $U$ de $\Omega$ com $\bar{x} \in \partial U$ e uma função $V: \Omega \rightarrow \mathbb{R}$ tal que $V<0$ em $U, V=0$ em $\partial U$ e $\dot{V}<0$ em $U$, então $\bar{x}$ é um ponto de equilíbrio instável de (4.1). Para provar a existência da solução assintótica a $\bar{x}$ precisamos fortalecer a hipótese sobre $V$. Mais precisamente

Teorema 4.3.1 Suponha que temos $\Omega, U, f$ e $V$ como acima e além disto, que

$$
\dot{V}(x)<0, \quad \forall x \in \partial U \backslash\{\bar{x}\}
$$

Então existe uma solução de (4.1) assintótica a $\bar{x}$ quando $t \rightarrow-\infty$.

Vamos provar uma forma mais forte deste resultado usando duas funções auxiliares, mais precisamente:

Teorema 4.3.2 Suponha que temos $\Omega, U, f$ e $\bar{x}$ como acima, e admita que existem duas funções $C^{1}, V$ e $W$, definidas em $\Omega$ tais que

(i) $\dot{V}(x)<0, \quad \forall x \in \bar{U} \backslash\{\bar{x}\}$,

(ii) $W(x)<0, \quad \forall x \in U$,

(iii) $W(x)=0, \quad \forall x \in \partial U$, 
(iv) $\dot{W}(x) \leq 0, \quad \forall x \in U$.

Então existe uma solução de (4.1) assintótica a $\bar{x}$ quando $t \rightarrow-\infty$.

Note que o Teorema 4.3.1 é um caso particular do Teorema 4.3.2 quando $V=W$, então é suficiente provar o último resultado.

\section{DEMONSTRAÇÃO}

Seja $\varepsilon>0$ tal que $B=\left\{x \in \mathbb{R}^{n}|\quad| x-\bar{x} \mid \leq \varepsilon\right\}$ está contido em $\Omega$ e denote por $U_{\varepsilon}$ o conjunto $B \cap \bar{U}$.

Considere um ponto $z \in U_{\varepsilon}^{\circ}$.

Provaremos primeiro que a solução $x_{z}$ de (4.1) tal que $x_{z}(0)=z$ deixa $U_{\varepsilon}^{\circ}$ através de $S=U_{\varepsilon} \cap \partial B$. Para isto, é suficiente mostrar que $x_{z}$ não pode permanecer em $U_{\varepsilon}$ no futuro, pois por $(i i),(i i i)$ e $(i v)$ temos que $x_{z}(t) \notin \partial U$ para cada $t>0$ em que esta solução é definida.

Suponha, por contradição, que $x_{z}$ permanece em $U_{\varepsilon}$ no futuro. Então usando a compacidade de $U_{\varepsilon}$ temos que $x_{z}$ é definida em $[0, \infty)$, e, como $W(\bar{x})=0$, vemos por $\left(\right.$ iii) e (iv) que há um $\delta>0$ tal que $\delta \leq\left|x_{z}(t)-\bar{x}\right| \leq \varepsilon$, para todo $t>0$.

Agora, aplicando $(i)$ vemos que $h=V \circ x_{z}$ é uma função $C^{1}$ definida em $[0, \infty)$ e há um $c>0$ tal que $\dot{h}(t)<-c$.

Isto é uma contradição com o fato de $h$ ser limitada em $[0, \infty)$.

Considere agora uma sequência $x_{n} \in U_{\varepsilon}^{\circ}$ convergente para $\bar{x}$, e denote por $\phi_{n}$ a solução de (4.1) com $\phi_{n}(0)=x_{n}$. Seja $y_{n}$ o ponto de $S$ em que $\phi_{n}$ deixa $U_{\varepsilon}^{\circ}$ pela primeira vez e $T_{n}>0$ o primeiro número positivo tal que $\phi_{n}\left(T_{n}\right)=y_{n}$.

Como $x_{n} \rightarrow \bar{x}$, que é um ponto de equilíbrio de (4.1), temos, pela dependência contínua das soluções de uma equação diferencial ordinária com respeito às condições iniciais, que $T_{n} \rightarrow \infty$ quando $n \rightarrow \infty$.

Podemos supor, pela compacidade de $S$, que $y_{n}$ converge para um ponto $\bar{y} \in S$. Seja $\phi$ a solução de $(4.1) \operatorname{com} \phi(0)=\bar{y}$.

Novamente pela dependência contínua das soluções com respeito às condições iniciais é fácil ver que existe um $T>0$ tal que $\phi(t) \in U_{\varepsilon}$ para $t \in[-T, 0)$; além disso provaremos que $\phi(t) \in U_{\varepsilon}$ para todo $t<0$ para o qual $\phi$ é definida.

De fato, suponha, por contradição, que isto é falso. Então existe um $\lambda<0$ tal que $\phi(\lambda) \notin U_{\varepsilon}$, e como $T_{n} \rightarrow \infty$, há um $n$ suficientemente grande tal que $T_{n}>2|\lambda|$. Então, pelo teorema da dependência contínua, $\phi_{n}$ deve deixar $U_{\varepsilon}$ em um instante $t_{n}$ tal que $0<t_{n}<T_{n}$; mas isto é uma contradição com a escolha de $y_{n}$ e $T_{n}$. Portanto, como $U_{\varepsilon}$ é compacto temos que $\phi$ é definida em $(-\infty, 0]$ e o conjunto alfa limite de esta solução, $\Lambda$, deve ser não vazio. 
Considere a função $C^{1}, g=V \circ \phi$, a qual é definida em $(-\infty, 0]$ e é limitada e estritamente decrescente. Então existe $\rho$ tal que $\lim _{t \rightarrow-\infty} g(t)=$ $\rho$.

Isto mostra que se $z \in \Lambda$ então $V(z)=\rho$, logo, pela invariância do conjunto alfa limite, temos $\dot{V}(z)=0$.

Agora, usando que $\Lambda \subset U_{\varepsilon}$ e $(i)$, concluimos que $\Lambda=\{\bar{x}\}$.

Fazemos um uso imediato deste resultado para provar:

Corolário 4.3.3 Seja $H=T+\Pi$ uma função hamiltoniana definida em $\Omega \times \mathbb{R}^{n}$, em que a energia potencial de classe $C^{2}, \Pi: \Omega \rightarrow \mathbb{R}$, tem um ponto crítico em $0 \in \Omega$, e seja $B=\{q \in \Omega|\quad| q \mid<\varepsilon\}$. Se existe uma componente conexa $C$ de $\Pi^{-1}((-\infty, 0))$ aderente à origem tal que $\langle\nabla \Pi(q), q\rangle<0$ para $q \in(\bar{C} \backslash\{0\}) \cap B$, então existe uma trajetória assintótica a 0.

DEMONSTRAÇÃo

É suficiente considerar

$$
U=\left\{(q, p) \in C \times \mathbb{R}^{n}|\quad\langle q, p\rangle>0, \quad|(q, p) \mid<\varepsilon \quad \text { e } \quad H(q, p)<0\right\}
$$

e funções $V, W: U \rightarrow \mathbb{R}$ definidas por $V(q, p)=-\langle q, p\rangle$ e $W(q, p)=$ $\langle q, p\rangle H(q, p)$.

Então, se lembramos que $T$ é homogênea de grau 2 em relação à variável $p$, uma aplicação do teorema de Euler para funções homogêneas e equação hamiltoniana mostra que

$$
\begin{aligned}
\dot{V}(q, p) & =-\left[2 T(q, p)-\left\langle\frac{\partial T}{\partial q}(q, p), q\right\rangle-\langle\nabla \Pi(q), q\rangle\right], \\
\dot{W}(q, p) & =\left[2 T(q, p)-\left\langle\frac{\partial T}{\partial q}(q, p), q\right\rangle-\langle\nabla \Pi(q), q\rangle\right] H(q, p) .
\end{aligned}
$$

Observe agora que podemos escolher $\varepsilon>0$ suficientemente pequeno para obter $R(q, p)=2 T(q, p)-\left\langle\frac{\partial T}{\partial q}(q, p), q\right\rangle>0$ se $|q|<\varepsilon$ e $p \neq 0$. De fato, esta é uma consequência de $R$ ser quadrática com respecto a $p$ e $R(0, p)=2 T(0, p)$ ser definida positiva.

Então vemos que as condições $(i i)$, $(i i i)$ e $(i v)$ do Teorema 4.3 .2 são verificadas.

Agora, se $(q, p) \in \bar{U} \backslash\{0\}$ temos $q \neq 0$, pois, caso contrário, $p \neq 0$ e $H(0, p)>0$, o que contraria $(0, p) \in \bar{U}$.

Isto mostra que $\dot{V}<0$ em $\bar{U} \backslash\{0\}$ e podemos aplicar o Teorema 4.3 .2 a fim de obter a nossa tese. 


\section{Bibliografia}

[B] Barone Neto; Jet-detectable Extrema; Proceeding A.M.S, (1984).

[FGT] R.S. Freire Jr., M.V.P. Garcia, F.A. Tal; Instability of equilibrium points of some Lagrangian systems; Journal of Differential Equations, 245, (2008), 490-504.

[G] Garcia M.V.; k-decidibilidade e estabilidade de Layapunov, RT-USP, (1992).

[GT] Manuel V.P. Garcia, Fábio A. Tal; Stability of equilibrium of conservative systems with two degrees of freedom; Journal of Differential Equations, 194, (2003), 364-381.

[LHR] N. Rouche, P. Habets, M. Laloy; Stability Theory by Liapunov`s Direct Method; Springer-Verlag New York, (1977).

[LP] M. Laloy, K. Peiffer; On the Instability of Equilibrium when the Potential has a Non-Strict Local Minimum; Arch.Rational Mech.Anal., 78, (1982), No 3, 213-222.

[M] John Milnor; Singular Points of Complex Hypersurfaces; Annals of Mathematics Studies, Princeton University Press.

[MN] Vinicio Moauro and Piero Negrini; On the Inversion of LagrangeDirichlet Theorem; Differential and Integral Equations, Volume 2, Number 4, October (1989), pp. 471-478.

[P] V. Palamodov; Stability of motion and algebraic geometry; Transl. Amer. Math. Soc. Ser. 2 Vol.168 (25) (1995) 5-20.

[S] Jorge Sotomayor; Lições de equações diferenciais ordinárias; Projeto Euclides, IMPA, (1979). 